\title{
Triangle-degrees in graphs and tetrahedron coverings in 3-graphs
}

\author{
Victor Falgas-Ravry ${ }^{1}$, Klas Markström ${ }^{1, *}$ and Yi Zhao ${ }^{2}$ \\ ${ }^{1}$ Department of Mathematics and Mathematical Statistics, Umeå Universitet, 90187 Umeå, Sweden. ${ }^{2}$ Department of \\ Mathematics and Statistics, Georgia State University, Atlanta, GA 30303, USA. \\ *Corresponding author. Email: klas.markstrom@umu.se
}

(Received 28 January 2019; revised 7 January 2020; first published online 9 September 2020)

\begin{abstract}
We investigate a covering problem in 3-uniform hypergraphs (3-graphs): Given a 3-graph $F$, what is $c_{1}(n, F)$, the least integer $d$ such that if $G$ is an $n$-vertex 3-graph with minimum vertex-degree $\delta_{1}(G)>d$ then every vertex of $G$ is contained in a copy of $F$ in $G$ ?

We asymptotically determine $c_{1}(n, F)$ when $F$ is the generalized triangle $K_{4}^{(3)-}$, and we give close to optimal bounds in the case where $F$ is the tetrahedron $K_{4}^{(3)}$ (the complete 3-graph on 4 vertices).

This latter problem turns out to be a special instance of the following problem for graphs: Given an $n$ vertex graph $G$ with $m>n^{2} / 4$ edges, what is the largest $t$ such that some vertex in $G$ must be contained in $t$ triangles? We give upper bound constructions for this problem that we conjecture are asymptotically tight. We prove our conjecture for tripartite graphs, and use flag algebra computations to give some evidence of its truth in the general case.
\end{abstract}

2010 MSC Codes: Primary 05C35; Secondary 05C65, 05D99

\section{Introduction}

Let $F$ be a graph with at least one edge. What is the maximum number of edges $\operatorname{ex}(n, F)$ an $n$ vertex graph can have if it does not contain a copy of $F$ as a subgraph? This is a classical question in extremal graph theory. If $F$ is a complete graph, then the exact answer is given by Turán's theorem [63], one of the cornerstones of extremal graph theory. For other graphs $F$, the value of $\operatorname{ex}(n, F)$ is determined up to an $o\left(n^{2}\right)$ error term by the celebrated Erdös-Stone theorem [17].

Ever since Turán's foundational result, there has been significant interest in obtaining similar 'Turán-type' results for $r$-uniform hypergraphs ( $r$-graphs), with $r \geqslant 3$. The extremal theory of hypergraphs has, however, turned out to be much harder, and even the fundamental question of determining the maximum number of edges in a 3-graph with no copy of the tetrahedron $K_{4}^{(3)}$ (the complete 3-graph on 4 vertices) remains open: it is the subject of a 70-year-old conjecture of Turán, and of an Erdös $\$ 1000$ prize. ${ }^{1}$ Most research efforts have focused on the case of 3-graphs,

\footnotetext{
${ }^{1}$ In fact, to earn this particular Erdős prize, it is sufficient to determine the limit $\lim _{n \rightarrow \infty} \operatorname{ex}\left(n, K_{t}^{(r)}\right) /\left(\begin{array}{l}n \\ r\end{array}\right)$ for any integers $t>r \geqslant 3$. 
where a small number of exact and asymptotic results are now known; see [3, 4, 12, 20, 24], as well as the surveys by Füredi [23], Sidorenko [61] and Keevash [34].

It is well known that the Turán problem for an $r$-graph $F$ is essentially equivalent to identifying the minimum vertex-degree required to guarantee the existence of a copy of $F$. More recently $[11,44,51]$, there has been interest in variants where one considers what minimum $i$-degree condition is required to guarantee the existence of a copy of $F$. Given an $i$-set $S \subseteq V(G)$ with $i \leqslant r$, its neighbourhood in $G$ is the collection

$$
\Gamma(S)=\Gamma_{G}(S):=\{T \subseteq V(G) \backslash S: S \cup T \in E(G)\}
$$

of $(r-i)$-sets $T$ whose union with $S$ makes an edge of $G$. The neighbourhood of $S$ defines an $(r-i)$-graph

$$
G_{S}:=\left(V(G) \backslash S, \Gamma_{G}(S)\right) \text {, }
$$

which is called the link graph of $S$. The degree of $S$ in $G$ is the size $\operatorname{deg}_{G}(S)=\operatorname{deg}(S):=|\Gamma(S)|$ of its neighbourhood. The minimum $i$-degree $\delta_{i}(G)$ of $G$ is the minimum of $\operatorname{deg}(S)$ over all $i$-subsets $S \subseteq V(G)$. In particular, the case $i=r-1$ has received particular attention; $\delta_{r-1}(G)$ is known as the minimum codegree of $G$, and a minimum codegree condition is the strongest single degree condition one can impose on an $r$-graph. Determining what minimum codegree forces the existence of a copy of a fixed $r$-graph $F$ is known as the codegree density problem [51]. A few results on the codegree density for various small 3-graphs are known; see [18, 19, 36, 49].

In a different direction, there has been significant recent research activity devoted to generalizing another foundational result in extremal graph theory. Let $F$ be a graph whose order divides $n$. What minimum degree condition is required to guarantee that a graph on $n$ vertices contains an F-tiling - a collection of $n / v(F)$ vertex-disjoint copies of $F$ ? In the case of complete graphs, this was answered by the celebrated Hajnal-Szemerédi theorem [27], which (under the guise of equitable colourings) has applications to scheduling problems. For a general graph $F$, the KühnOsthus theorem [40] determines the minimum degree threshold for F-tilings up to a constant additive error.

There has been a growing interest in determining analogous tiling thresholds in $r$-graphs for $r \geqslant 3$; see the surveys by Rödl and Ruciński [59] and Zhao [64] devoted to the subject. In an effort to generalize Dirac's theorem on Hamilton cycles to hypergraphs, Rödl, Ruciński and Szemerédi [60] determined the minimum codegree threshold for the existence of a perfect matching in $r$ graphs for $r \geqslant 3$. The paper also introduced the hugely influential absorption method, which has been used as a key ingredient in many of the results in the area obtained since. Beyond perfect matchings, codegree tiling thresholds have now been determined for a number of small 3-graphs, including $K_{4}^{(3)}[35,45], K_{4}^{(3)-}[30,43]$ and $K_{4}^{(3)--}\left(K_{4}^{(3)}\right.$ with two edges removed $)[10,39]$. In addition, the codegree tiling thresholds for $r$-partite $r$-graphs have been studied recently $[9,25$, $26,29,52]$

For minimum vertex-degree tiling thresholds, fewer results are known. The vertex-degree thresholds for perfect matchings were determined for 3-graphs by Han, Person and Schacht [28] (asymptotically) and by Kühn, Osthus and Treglown [41] and Khan [38] (exactly). Han and Zhao [32] determined the vertex-degree tiling threshold for $K_{4}^{(3)--}$, while Han, Zang and Zhao [31] asymptotically determined the vertex-degree tiling threshold for all complete 3-partite 3-graphs.

As a key part of their argument, Han, Zang and Zhao considered a certain 3-graph covering problem and showed that it was distinct from the corresponding Turán-type existence problem. This stands in contrast to the situation for ordinary graphs, where existence and covering thresholds essentially coincide. Given an $r$-graph $F$, Falgas-Ravry and Zhao [21] introduced the notion of an $F$-covering, which is intermediate between that of the existence of a single copy of $F$ and the existence of an $F$-tiling. 
We say that an $r$-graph $G$ has an $F$-covering if every vertex in $G$ is contained in a copy of $F$ in $G$. Equivalently, an $F$-covering of $G$ is a collection $C$ of copies $F$ whose union covers all of $V(G)$. For every positive integer $i \leqslant r-1$, the $i$-degree $F$-covering threshold is the function

$$
c_{i}(n, F):=\max \left\{\delta_{i}(G): v(G)=n, G \text { has no } F \text {-covering }\right\} .
$$

We further let the $i$-degree $F$-covering density be the limit ${ }^{2}$

$$
c_{i}(F):=\lim _{n \rightarrow \infty} c_{i}(n, F) /\left(\begin{array}{c}
n-i \\
r-i
\end{array}\right) .
$$

Let $K_{t}^{(r)}$ denote the complete $r$-graph on $t$ vertices and let $K_{t}^{(r)-}$ denote the $r$-graph obtained by removing one edge from $K_{t}^{(r)}$. A tight $r$-uniform $t$-cycle $C_{t}^{(r)}$ is an $r$-graph with a cyclic ordering of its $t$ vertices such that every $r$ consecutive vertices form an edge under this ordering. Falgas-Ravry and Zhao [21] determined $c_{2}(F)$, where $F$ is $K_{4}^{(3)}, K_{4}^{(3)-}, K_{5}^{(3)-}$ and $C_{5}^{(3)}$. Han, Lo and SanhuezaMatamala [29] determined $c_{r-1}\left(C_{t}^{(r)}\right)$ for all $r \geqslant 3$ and $t>2 r^{2}$.

In this paper we investigate $c_{1}(n, F)$ and $c_{1}(F)$ for various 3 -graphs $F$. We first consider $K_{4}^{(3)-}$. Let $f_{n}(d)$ be the function

$$
f_{n}(d):=\left(\begin{array}{c}
n-2 \\
2
\end{array}\right)+d-d(d-1)-\left(\begin{array}{l}
d \\
2
\end{array}\right)=\frac{1}{2}\left(n^{2}-5 n+6-3 d^{2}+5 d\right) .
$$

Observe that for fixed $n, f_{n}(d)$ is a decreasing function of $d$ over the interval $[1, n-2]$. On the other hand $((n-1) / 2) d$ is an increasing function of $d$, so there exists a unique $d_{\star}=d_{\star}(n)$ such that $((n-1) / 2) d_{\star}=f\left(n, d_{\star}\right)$, namely

$$
d_{\star}=\frac{1}{6}\left(\sqrt{13 n^{2}-72 n+108}-n+6\right)=\frac{\sqrt{13}-1}{6} n+O(1) .
$$

Theorem 1.1. For all odd integer $n$,

$$
\frac{n-1}{2}\left\lfloor d_{\star}\right\rfloor \leqslant c_{1}\left(n, K_{4}^{(3)-}\right) \leqslant\left\lfloor\frac{n-1}{2} d_{\star}\right\rfloor .
$$

In particular,

$$
c_{1}\left(K_{4}^{(3)-}\right)=\frac{\sqrt{13}-1}{6}=0.4342 \ldots
$$

The upper and lower bounds on $c_{1}(n, F)$ in Theorem 1.1 differ by less than $n / 2$. However, it seems that much more work will be needed to determine $c_{1}(n, F)$ exactly. As a first step in this direction, we prove the following stability theorem characterizing near-extremal configurations. Let $c_{\star}=(\sqrt{13}-1) / 6$.

Theorem 1.2. For every $\varepsilon>0$, there exists $\delta>0$ and $n_{0} \in \mathbb{N}$ such that the following holds: for every $n \geqslant n_{0}$, if $H$ is a 3-graph on $n+1$ vertices with minimum vertex-degree at least $\left(c_{\star}-\delta\right)\left(n^{2} / 2\right)$ and $x \in V(H)$ is not covered by a copy of $K_{4}^{(3)-}$ in $H$, then the link graph $H_{x}$ can be made bipartite by removing at most $\varepsilon n^{2}$ edges.

Next we consider $K_{4}^{(3)}$.

\footnotetext{
${ }^{2}$ This limit can be shown to exist: see [21, footnote 1$]$.
} 


\section{Theorem 1.3.}

$$
\frac{19}{27}=0.7037 \ldots \leqslant c_{1}\left(K_{4}^{(3)}\right) \leqslant \frac{19}{27}+7.4 \times 10^{-9}
$$

The upper bound was derived from the flag algebra method. We believe that the lower bound is tight. As we show in Section 2.3, the problem of determining $c_{1}\left(K_{4}^{(3)}\right)$ is equivalent to (a special case of) a problem about triangle-degrees in graphs.

Given a graph $G$, the triangle-degree of a vertex $x \in V(G)$ is the number of triangles that contains $x$. The well-studied Rademacher-Turán problem concerns the smallest average triangledegree for all graphs with a given edge density (the edge density $\rho(G)$ is defined as $e(G) /\left(\begin{array}{c}v(G) \\ 2\end{array}\right)$ ). This problem attracted significant attention (see $[5,14,22,46,47])$ until it was resolved asymptotically by Razborov [56] using the framework of his newly developed theory of flag algebras. Different proofs expressed in the language of weighted graphs were later found by Nikiforov [53] and by Reiher [58] (who generalized Razborov's result to cliques of order 4 and of arbitrary order $t$, respectively).

Let $t_{\max }(G)$ denote the maximum triangle-degree in $G$. (This is related to but different from the well-studied book number, which is the maximum number of triangles containing a fixed edge of $G$; see the discussion in Section 4 for details.) For $\rho \in[0,1]$, we define

$$
\tau(\rho):=\liminf _{n \rightarrow \infty} \min \left\{t_{\max }(G) /\left(\begin{array}{c}
n-1 \\
2
\end{array}\right): v(G)=n, \rho(G) \geqslant \rho\right\},
$$

which is the asymptotically smallest maximum scaled triangle-degree in a graph with edge density $\rho$. We derive the following upper bounds for $\tau(\rho)$ and conjecture that they are tight. If Conjecture 1.7 holds, then $c_{1}\left(K_{4}^{(3)}\right)=19 / 27$ (see Proposition 3.1).

Theorem 1.4. Suppose

$$
\rho \in\left[\frac{r-1}{r}, \frac{r}{r+1}\right] \text { for some } r \in \mathbb{N} \text {. }
$$

Then

$$
\tau(\rho) \leqslant \begin{cases}\frac{(r-1)(r-2)}{r^{2}}+\frac{3(r-1)}{r}\left(\rho-\frac{r-1}{r}\right) & \text { if } \frac{r-1}{r} \leqslant \rho \leqslant \frac{r}{r+1}-\frac{1}{3 r(r+1)}, \\ \frac{r(r-1)}{(r+1)^{2}}-\frac{3(r-1)}{r+1}\left(\frac{r}{r+1}-\rho\right) & \text { if } \frac{r}{r+1}-\frac{1}{3 r(r+1)} \leqslant \rho \leqslant \frac{r}{r+1} .\end{cases}
$$

The constructions underpinning Theorem 1.4 are very different from the extremal ones for the Rademacher-Turán problem, and are discussed in more detail in Section 3.

Construction 1.5. (lower interval construction). Let

$$
\rho \in\left[\frac{r-1}{r}, \frac{r}{r+1}-\frac{1}{3 r(r+1)}\right] \text { for some } r \in \mathbb{N} .
$$

Suppose $n \in \mathbb{N}$ is divisible by $2 r$. Consider a balanced complete r-partite graph on $[n]$ with parts $V_{1}, \ldots, V_{r}$. Add inside each $V_{i}$ an arbitrary d-regular triangle-free graph $H_{i}$, where

$$
d=\left\lfloor\left(\rho-\frac{r-1}{r}\right) n\right\rfloor
$$


Such triangle-free graphs exist since

$$
d \leqslant \frac{2}{3(r+1)} \frac{n}{r}
$$

(by our upper bound on $\rho$ ), which is less than $n /(2 r)$ (so one could take $H_{i}$ to be a balanced bipartite graph, for example). The resulting graph is $\lfloor\rho n\rfloor$-regular. We let $\mathcal{G}_{\rho, n}^{u}$ denote the family of all graphs that can be constructed in this way.

Construction 1.6. (upper interval construction). Let

$$
\rho \in\left[\frac{r}{r+1}-\frac{1}{3 r(r+1)}, \frac{r}{r+1}\right] \text { for some } r \in \mathbb{N} .
$$

Suppose $n \in \mathbb{N}$ is divisible by $2(r+1)$. Consider a balanced complete $(r+1)$-partite graph on $[n]$ with parts $V_{1}, \ldots, V_{r+1}$. Equally divide each $V_{i}$ into $V_{i}^{\prime}$ and $V_{i}^{\prime \prime}$. Let $\phi:[r+1] \rightarrow[r+1]$ be any bijection with the property that $\phi(i) \neq i$ for all $i \in[r+1]$ (any permutation of $[r+1]$ with no fixed point will do). Now for every $i \in[r+1]$, replace the complete bipartite graph between $V_{i}^{\prime}$ and $V_{\phi(i)}^{\prime \prime}$ with an arbitrary d-regular bipartite subgraph $H_{i}$, where

$$
d=\left\lceil\left(\rho-\frac{r}{r+1}+\frac{1}{2(r+1)}\right) n\right\rceil .
$$

The resulting graph is $\lceil\rho n\rceil$-regular. We let $\mathcal{G}_{\rho, n}^{d}$ denote the family of all graphs that can be constructed in this way.

Conjecture 1.7. The upper bounds on $\tau(\rho)$ given in Theorem 1.4 are tight for every $\rho \in[0,1]$.

We use flag algebra computations to show that the upper bounds from Conjecture 1.7 are not far from optimal when $\rho \in[1 / 2,2 / 3]$ (see Theorem 3.6).

Following on a beautiful result of Bondy, Shen, Thomassé and Thomassen [7] on a tripartite version of Mantel's theorem, Baber, Johnson and Talbot [2] gave a tripartite analogue of Razborov's triangle-density result. In a similar spirit, we prove that Conjecture 1.7 holds for tripartite graphs. Note that a tripartite graph on $n$ vertices can have between 0 and $n^{2} / 3$ edges.

Theorem 1.8. Let $G$ be a tripartite graph on $n$ vertices. Then

$$
t_{\max }(G) \geqslant \begin{cases}\frac{3}{2}\left(e(G)-\frac{n^{2}}{4}\right) & \text { if } \frac{e(G)}{n^{2}}<\frac{3}{10} \\ e(G)-\frac{2}{9} n^{2} & \text { if } \frac{3}{10} \leqslant \frac{e(G)}{n^{2}} \leqslant \frac{1}{3} .\end{cases}
$$

Structure of the paper. In Section 2 we prove Theorems 1.1-1.3 along with bounds for $c_{1}\left(C_{5}^{(3)}\right)$ and $c_{1}\left(K_{t}^{(3)}\right)$ for $t \geqslant 5$. In Section 3 we prove Theorems 1.4 and 1.8 , and give flag algebra bounds on $\tau(\rho)$. We end the paper in Section 4 with a discussion of book numbers in graphs and a comparison of known results and conjectures on minimal triangle density, triangle-degree and book number as functions of edge density.

Notation. We use standard graph and hypergraph theory notation throughout the paper. In addition, we use $[n]$ to denote the set $\{1,2, \ldots, n\}$ and $S^{(r)}$ to denote the collection of all $r$-subsets of a set $S$. Where there is no risk of confusion, we identify hypergraphs with their edge-sets. 


\section{Covering in 3-graphs}

\subsection{Proof of Theorem 1.1}

Recall that $K_{4}^{(3)-}$ is the (unique up to isomorphism) 3-graph on 4 vertices spanning 3 edges, also known as the generalized triangle. In this subsection we prove Theorem 1.1.

Proof of Theorem 1.1, lower bound. Let $n$ be odd, and let $d=\left\lfloor d_{\star}\right\rfloor \leqslant(n-1) / 2$. We construct a 3 -graph $H$ on $n$ vertices as follows. Set aside a vertex $v_{\star}$, and let $A \sqcup B$ be a bipartition of $V(H) \backslash$ $\left\{v_{\star}\right\}$ into two sets of equal size. Let $G$ be an arbitrary $d$-regular bipartite graph with partition $A \sqcup B$. Now let $H$ be the 3-graph whose 3-edges are the union of the triples $\left\{v_{\star} x y: x y \in E(G)\right\}$ together with all the triples of vertices from $A \cup B$ inducing at most one edge in $G$.

Clearly, for every triple of vertices $S \subseteq A \cup B, S \cup\left\{v_{\star}\right\}$ induces at most two edges of $H$ and $v_{\star}$ is not contained in any copy of $K_{4}^{(3)-}$. Thus $c_{1}\left(n, K_{4}^{(3)-}\right) \geqslant \delta_{1}(H)$. This latter quantity is easily calculated: the degree of $v_{\star}$ in $H$ is $((n-1) / 2) d$. For any $a \in A$, there are exactly $d(d-1)$ pairs $\left(a^{\prime}, b\right) \in A \times B$ such that both $a^{\prime} b$ and $a b$ lie in $G$, and exactly $\left(\begin{array}{l}d \\ 2\end{array}\right)$ pairs $\left(b, b^{\prime}\right) \in B^{(2)}$ such that both $a b$ and $a b^{\prime}$ lie in $G$; such pairs are the only pairs from $((A \backslash\{a\}) \cup B)^{(2)}$ that do not form an edge of $H$ with $a$. In addition, there are exactly $d$ edges of $H$ containing the pair $a v_{\star}$. Thus the degree of $a$ in $H$ is

$$
\operatorname{deg}(a)=\left(\begin{array}{c}
n-2 \\
2
\end{array}\right)-d(d-1)-\left(\begin{array}{l}
d \\
2
\end{array}\right)+d=f_{n}(d) .
$$

By symmetry, the degree of any vertex in $B$ is also $f_{n}(d)$. Thus

$$
\delta_{1}(H)=\min \left(\frac{n-1}{2} d, f_{n}(d)\right)=\frac{n-1}{2} d
$$

because $d \leqslant d_{\star}$. Since $H$ has no $K_{4}^{(3)-}$-covering, it follows that

$$
c_{1}\left(n, K_{4}^{(3)-}\right) \geqslant \frac{n-1}{2}\left\lfloor d_{\star}\right\rfloor .
$$

Proof of Theorem 1.1, upper bound. Suppose $H$ is a 3-graph on $n$ vertices with $\delta_{1}(H)=$ $((n-1) / 2) d$ and no copy of $K_{4}^{(3)-}$ covering a vertex $x$ (here $n$ is not necessarily odd). We shall show that $\delta_{1}(H) \leqslant((n-1) / 2) d_{\star}$. Note that the link graph $H_{x}$ of $x$ is triangle-free. Furthermore, $v_{1} v_{2} v_{3} \notin E(H)$ for any triple $v_{1} v_{2} v_{3}$ spanning two edges in $H_{x}$. Let $F(v)$ denote the collection of pairs $v_{2} v_{3}$ such that $v v_{2} v_{3}$ induces two edges in $H_{x}$. We know that $v v_{2} v_{3} \notin E(H)$ for every $v_{2} v_{3} \in F(v)$. Observe that $F(v)$ consists of all pairs $v_{2} v_{3}$, where either $v_{2}, v_{3} \in \Gamma(x, v)$ or $v_{2} v_{3} \in H_{x}$ and exactly one of $v_{2}, v_{3}$ is in $\Gamma(x, v)$.

Counting non-edges of $H$ over all $v \in V \backslash\{x\}$, we thus have

$$
\begin{aligned}
& \sum_{v \in V \backslash\{x\}}\left(\left(\begin{array}{c}
n-1 \\
2
\end{array}\right)-\operatorname{deg}(v)\right) \\
& \geqslant \sum_{v \in V \backslash\{x\}} n-2-\operatorname{deg}(x, v)+|F(v)| \\
& \geqslant \sum_{v \in V \backslash\{x\}}\left(n-2-\operatorname{deg}(x, v)+\left(\begin{array}{c}
\operatorname{deg}(x, v) \\
2
\end{array}\right)+\sum_{v_{2} \in \Gamma(x, v)}\left(\operatorname{deg}\left(x, v_{2}\right)-1\right)\right) \\
& =(n-1)(n-2)+\sum_{v \in V \backslash\{x\}} \frac{1}{2}\left(3(\operatorname{deg}(x, v))^{2}-5 \operatorname{deg}(x, v)\right)
\end{aligned}
$$




$$
\begin{aligned}
& \geqslant(n-1)(n-2)+\frac{n-1}{2}\left(3 d^{2}-5 d\right) \\
& =(n-1)\left(n-2+\frac{3 d^{2}-5 d}{2}\right) .
\end{aligned}
$$

where in the last line we used Jensen's inequality and our minimum degree assumption deg $(x) \geqslant$ $((n-1) / 2) d$. By averaging, there exists a vertex $v \in v \in V \backslash\{x\}$ with

$$
\operatorname{deg}(v) \leqslant\left(\begin{array}{c}
n-1 \\
2
\end{array}\right)-n+2-\frac{3 d^{2}-5 d}{2}=f_{n}(d) .
$$

Applying our minimum degree assumption $\operatorname{deg}(v) \geqslant((n-1) / 2) d$ yields $((n-1) / 2) d \leqslant f_{n}(d)$ and hence $d \leqslant d_{\star}$. Thus $\delta_{1}(H) \leqslant((n-1) / 2) d_{\star}$ as claimed.

\subsection{Proof of Theorem 1.2}

Our proof will make use of a consequence of Karamata's inequality. Let $a_{n} \geqslant a_{n-1} \geqslant \cdots \geqslant a_{1}$ and $b_{n} \geqslant b_{n-1} \geqslant \cdots \geqslant b_{1}$ be real numbers. We say that $\mathbf{a}=\left(a_{n}, \ldots, a_{1}\right)$ majorizes $\mathbf{b}=\left(b_{n}, \ldots, b_{1}\right)$ if $\sum_{i \geqslant k} a_{i} \geqslant \sum_{i \geqslant k} b_{i}$ for all $1 \leqslant k \leqslant n$, with equality attained in the case $k=1$. Karamata's inequality states that, if $\mathbf{a}$ majorizes $\mathbf{b}$ and $f$ is a convex function, then $\sum_{i} f\left(a_{i}\right) \geqslant \sum_{i} f\left(b_{i}\right)$.

Lemma 2.1. Suppose $f: \mathbb{R} \rightarrow \mathbb{R}$ is a convex function. Let $a_{1} \leqslant a_{2} \leqslant \cdots \leqslant a_{n}$ be real numbers such that $\sum_{i} a_{i}=\bar{a} n$, and let $\eta>0$. Set $\mathcal{B}:=\left\{i: a_{i} \leqslant(1-\eta) \bar{a}\right\}$. Then

$$
\sum_{i} f\left(a_{i}\right) \geqslant|\mathcal{B}| \cdot f((1-\eta) \bar{a})+(n-|\mathcal{B}|) \cdot f\left(\left(1+\frac{\eta|\mathcal{B}|}{n-|\mathcal{B}|}\right) \bar{a}\right) .
$$

Proof. Since $\eta>0$, our assumption on $\sum_{i} a_{i}$ tells us that $[n] \backslash \mathcal{B} \neq \emptyset$. If $\mathcal{B}=\emptyset$, then the claimed inequality is just Jensen's inequality. So assume $\mathcal{B}$ is non-empty and set $|\mathcal{B}|=\beta$ for some $\beta>0$.

Let $a_{1}^{\prime}, a_{2}^{\prime}, \ldots, a_{n}^{\prime}$ be given by

$$
a_{i}^{\prime}= \begin{cases}(1-\eta) \bar{a} & \text { if } i \in[\beta n], \\ \left(1+\frac{\eta \beta}{1-\beta}\right) \bar{a} & \text { if } i \in[n] \backslash[\beta n] .\end{cases}
$$

Observe that $\sum_{i} a_{i}^{\prime}=\sum_{i} a_{i}=\bar{a} n$. Setting

$$
x=\frac{1}{|\mathcal{B}|} \sum_{i \in \mathcal{B}} a_{i} \quad \text { and } \quad y=\frac{1}{n-|\mathcal{B}|} \sum_{i \in[n] \backslash \mathcal{B}} a_{i},
$$

we have

$$
x \leqslant(1-\eta) \bar{a}<\left(1+\frac{\eta \beta}{1-\beta}\right) \bar{a} \leqslant \frac{\bar{a}-\beta x}{1-\beta}=y .
$$

It follows readily that the $n$-tuple $\left(a_{n}, a_{n-1}, a_{n-2}, \ldots, a_{1}\right)$ majorizes $\left(a_{n}^{\prime}, a_{n-1}^{\prime}, a_{n-2}^{\prime}, \ldots, a_{1}^{\prime}\right)$. Applying Karamata's inequality to the convex function $f$, we obtain

$$
\sum_{i} f\left(a_{i}\right) \geqslant \sum_{i} f\left(a_{i}^{\prime}\right)=\beta n \cdot f((1-\eta) \bar{a})+(1-\beta) n \cdot f\left(\left(1+\frac{\eta \beta}{1-\beta}\right) \bar{a}\right) .
$$

Another ingredient in the proof of Theorem 1.2 is a classical result of Andrásfai, Erdős and Sós. 
Theorem 2.2. (Andrásfai, Erdös and Sós [1]). Let $G$ be a triangle-free graph on $n$ vertices with minimum degree $\delta(G)>2 n / 5$. Then $G$ is bipartite.

With these two preparatory results in hand, the proof of Theorem 1.2 is straightforward. We first use Lemma 2.1 to show that the overwhelming majority of vertices in the link graph $H_{x}$ have degree much larger than $2 n / 5$, whereupon we deduce from the Andrásfai-Erdős-Sós theorem that $H_{x}$ is almost bipartite.

Proof of Theorem 1.2. Recall

$$
c_{\star}=\frac{\sqrt{13}-1}{6}=0.43 \ldots>\frac{2}{5} .
$$

Fix $\varepsilon>0$. Without loss of generality, assume that $\varepsilon<1 / 3\left(c_{\star}-2 / 5\right)$. Pick

$$
0<\eta<\frac{1}{3 c_{\star}}\left(c_{\star}-\frac{2}{5}\right) \text { and } \delta>0
$$

such that

$$
\delta<\frac{1}{3}\left(c_{\star}-\frac{2}{5}\right)<\frac{c_{\star}}{6} \quad \text { and } \quad\left(\frac{1+6 c_{\star}}{2 c_{\star}^{2} \eta^{2}}\right) \delta<\frac{\varepsilon}{2}
$$

both hold.

Let $H$ be a 3-graph with

$$
v(H)=n+1, \quad \delta_{1}(H) \geqslant\left(c_{\star}-\delta\right) \frac{n^{2}}{2} .
$$

Suppose $x$ is a vertex in $H$ not covered by any copy of $K_{4}^{(3)-}$. Without loss of generality, assume $V(H)=[n] \cup\{x\}$. By the vertex-degree assumption, $e\left(H_{x}\right)=c n^{2}$ for some $c \geqslant c_{\star}-\delta$. Let

$$
\mathcal{B}=\{y \in V(H): \operatorname{deg}(x y) \leqslant c(1-\eta) n\}
$$

be the collection of vertices in $H$ whose codegree with $x$ is smaller than average by a multiplicative factor of $(1-\eta)$. Set $|\mathcal{B}|=\beta n$.

Since $x$ is not covered by a copy of $K_{4}^{(3)-}$ in $H$, the following hold:

(i) $H_{x}$ is triangle-free,

(ii) for every triple of vertices $\left\{y_{1}, y_{2}, y_{3}\right\}$ inducing two edges in $H_{x}$, the 3 -edge $y_{1} y_{2} y_{3}$ is missing from $E(H)$.

Property (i) implies that for every $y \in[n]$, the neighbourhood $\Gamma(x y)$ is an independent set in $H_{x}$, while property (ii) implies that for every $z, z^{\prime} \in H_{x y}$ and every $w \in H_{x z}$, the 3-edges $z z^{\prime} y$ and $z w y$ are both missing from $E(H)$. In particular, for every $y \in[n]$ we have

$$
\left(1-c_{\star}+\delta\right) \frac{n^{2}}{2}>\left(\begin{array}{l}
n \\
2
\end{array}\right)-e\left(H_{y}\right) \geqslant\left(\begin{array}{c}
\left|H_{x y}\right| \\
2
\end{array}\right)+\sum_{z \in H_{x y}}\left(\left|H_{x z}\right|-1\right) .
$$

Summing this inequality over all $y \in[n]$ and using the fact that

$$
\sum_{y \in[n]} \sum_{z \in H_{x y}}\left(\left|H_{x z}\right|-1\right)=2 \sum_{y \in[n]}\left(\begin{array}{c}
\left|H_{x y}\right| \\
2
\end{array}\right)
$$


we get

$$
\left(1-c_{\star}+\delta\right) \frac{n^{3}}{2}>\sum_{y \in[n]} 3\left(\begin{array}{c}
\left|H_{x y}\right| \\
2
\end{array}\right) .
$$

Since the function $f(t)=\left(\begin{array}{l}t \\ 2\end{array}\right)$ is convex and $\sum_{y \in[n]}\left|H_{x y}\right|=2\left|H_{x}\right|=c n^{2}$, we can apply Lemma 2.1 to bound from below the right-hand side of (2.3) by

$$
\begin{aligned}
& 3\left(\beta n\left(\begin{array}{c}
c(1-\eta) n \\
2
\end{array}\right)+(1-\beta) n\left(\begin{array}{c}
\frac{c n^{2}-\beta n \cdot c(1-\eta) n}{(1-\beta) n} \\
2
\end{array}\right)\right) \\
& \quad=\frac{3 c^{2}}{2}\left(\beta(1-\eta)^{2}+\frac{(1-\beta(1-\eta))^{2}}{1-\beta}\right) n^{3}+O\left(n^{2}\right) .
\end{aligned}
$$

Inserting this inequality back into (2.3), dividing through by $n^{3}$ and using $c \geqslant c_{\star}-\delta$ yields

$$
\begin{aligned}
1-c_{\star}+\delta & \geqslant 3\left(c_{\star}-\delta\right)^{2}\left(\beta(1-\eta)^{2}+\frac{(1-\beta(1-\eta))^{2}}{1-\beta}\right)+O\left(n^{-1}\right) \\
& =3\left(c_{\star}-\delta\right)^{2}\left(1+\eta^{2} \beta+\frac{\eta^{2} \beta^{2}}{1-\beta}\right)+O\left(n^{-1}\right) \\
& >\left(3 c_{\star}^{2}-6 \delta c_{\star}\right)\left(1+\eta^{2} \beta\right)+O\left(n^{-1}\right) \\
& \geqslant 3 c_{\star}^{2}-6 \delta c_{\star}+2 c_{\star}^{2} \eta^{2} \beta+O\left(n^{-1}\right),
\end{aligned}
$$

where the last inequality holds because our choice of $\delta$ in (2.2) ensures $\delta<c_{\star} / 6$. Note that $c_{\star}$ satisfies $1-c_{\star}=3 c_{\star}^{2}$. Rearranging terms in inequality (2.5) gives

$$
\left(1+6 c_{\star}\right) \delta>2 c_{\star}^{2} \eta^{2} \beta+O\left(n^{-1}\right) .
$$

By the second part of (2.2) and the assumption that $n$ is sufficiently large, we have

$$
\beta<\left(\frac{1+6 c_{\star}}{2 c_{\star}^{2} \eta^{2}}\right) \delta+O\left(n^{-1}\right)<\frac{\varepsilon}{2}+O\left(n^{-1}\right)<\varepsilon
$$

and $|\mathcal{B}|=\beta n<\varepsilon n$. Remove from $H_{x}$ all vertices from $\mathcal{B}$. By the definitions of $\delta, \eta, \varepsilon$, the resulting triangle-free graph $G$ has at most $n$ vertices and minimum degree at least

$$
c(1-\eta) n-\varepsilon n \geqslant\left(c_{\star}-\delta\right)(1-\eta) n-\varepsilon n>\left(c_{\star}-\eta c_{\star}-\delta-\varepsilon\right) n>\frac{2}{5} n .
$$

By Theorem 2.2, $G$ is bipartite. Since we removed only at most $\varepsilon n$ vertices from $H_{x}$ to obtain $G$, it follows that $H_{x}$ can be made bipartite by removing at most $\varepsilon n^{2}$ edges, as claimed. This concludes the proof of Theorem 1.2.

\subsection{Proof of Theorem 1.3}

Given an $r$-graph $G$, write $t_{G}(x)$ for the number of copies of $K_{r+1}^{(r)}$ in $G$ that cover $x$.

Proposition 2.3. There exists an $r$-graph $H$ on $n+1$ vertices with minimum vertex-degree $\delta_{1}(H) \geqslant$ $\alpha\left(\begin{array}{c}n-1 \\ r-1\end{array}\right)$ and no $K_{r+1}^{(r)}$-covering if and only if there exists an $(r-1)$-graph $G$ on $n$ vertices with at least $\alpha\left(\begin{array}{c}n-1 \\ r-1\end{array}\right)$ edges such that, for every vertex $x \in V(G)$,

$$
t_{G}(x)-\operatorname{deg}_{G}(x) \leqslant(1-\alpha)\left(\begin{array}{c}
n-1 \\
r-1
\end{array}\right) .
$$


Proof. In one direction, let $H$ be an r-graph on $n+1$ vertices with minimum degree $\alpha\left(\begin{array}{c}n-1 \\ r-1\end{array}\right)$. Suppose $v_{\star}$ is not covered by any $K_{r+1}^{(r)}$ in $H$. By the minimum degree condition on $v_{\star}$, the $(r-1)$-uniform link graph $G=H_{v_{\star}}$ contains at least $\alpha\left(\begin{array}{c}n-1 \\ r-1\end{array}\right)$ edges. Also, every copy of $K_{r}^{(r-1)}$ in the $(r-1)$-graph $G$ must be a non-edge in the r-graph $H$, else together with $v_{\star}$ it would make a copy of $K_{r+1}^{(r)}$ in $H$ covering $v_{\star}$. The minimum degree condition in $H$ then implies that, for every vertex $x$ in the $n$-vertex $(r-1)$-graph $G$,

$$
\alpha\left(\begin{array}{l}
n-1 \\
r-1
\end{array}\right) \leqslant \operatorname{deg}_{H}(x) \leqslant\left(\begin{array}{l}
n-1 \\
r-1
\end{array}\right)+\operatorname{deg}_{G}(x)-t_{G}(x),
$$

implying $t_{G}(x)-\operatorname{deg}_{G}(x) \leqslant(1-\alpha)\left(\begin{array}{c}n-1 \\ 2\end{array}\right)$ as desired.

In the other direction, let $G$ be an $(r-1)$-graph on $n$ vertices with at least $\alpha\left(\begin{array}{l}n-1 \\ r-1\end{array}\right)$ edges such that $t_{G}(x)-\operatorname{deg}_{G}(x) \leqslant(1-\alpha)\left(\begin{array}{l}n-1 \\ r-1\end{array}\right)$ for all $x \in V(G)$. We add a new vertex $v_{\star}$ to $G$ and define an $r$-graph $H$ on $V(G) \sqcup\left\{v_{\star}\right\}$ by setting the link graph of $v_{\star}$ to be equal to $G$ and adding in as edges all $r$ sets from $V(G)^{(r)}$ that do not induce a copy of $K_{r}^{(r-1)}$ in $G$. This yields an r-graph on $n+1$ vertices in which $v_{\star}$ is not covered by a copy of $K_{r+1}^{(r)}, \operatorname{deg}_{H}\left(v_{\star}\right)=e(G) \geqslant \alpha\left(\begin{array}{c}n-1 \\ r-1\end{array}\right)$, and for every $x \in V(H) \backslash\left\{v_{\star}\right\}$,

$$
\operatorname{deg}_{H}(x)=\left(\begin{array}{l}
n-1 \\
r-1
\end{array}\right)-t_{G}(x)+\operatorname{deg}_{G}(x) \geqslant \alpha\left(\begin{array}{l}
n-1 \\
r-1
\end{array}\right),
$$

so $\delta_{1}(H) \geqslant \alpha\left(\begin{array}{l}n-1 \\ r-1\end{array}\right)$ as desired.

Corollary 2.4. For any $r \in \mathbb{N}$, the 1 -degree covering density $c_{1}\left(K_{r+1}^{(r)}\right)$ is the least $\alpha>0$ such that, if $G$ is an $(r-1)$-graph on $n$ vertices with at least $\alpha\left(\begin{array}{c}n \\ r-1\end{array}\right)$ edges, then there is a vertex $x \in G$ contained in $t_{G}(x) \geqslant(1-\alpha+o(1))\left(\begin{array}{c}n-1 \\ r-1\end{array}\right)$ copies of $K_{r}^{(r-1)}$ in $G$.

Proof of Theorem 1.3, lower bound. Suppose $3 \mid n$ and partition $[n]$ into three sets $V_{1}, V_{2}, V_{3}$ of size $n / 3$. Further partition each $V_{i}$ into two sets $V_{i, 1}$ and $V_{i, 2}$ of size $n / 6$. Now let $G$ be the 2-graph on $[n]$ obtained by putting in all edges of the form $V_{i} V_{j}$, with $1 \leqslant i<j \leqslant 3$ and adding for each $i \in$ [3] an arbitrary $n / 27$-regular bipartite graph with partition $V_{i, 1} \sqcup V_{i, 2}$. An easy calculation shows that $G$ is both regular and triangle-degree regular, with every vertex $x$ satisfying $\operatorname{deg}(x)=19 n / 27$ and $t(x)=4 n^{2} / 27$. We thus have

$$
t(x)-\operatorname{deg}(x)=\frac{8}{27}\left(\begin{array}{c}
n-1 \\
2
\end{array}\right)+O(n) .
$$

It follows from Proposition 2.3 that there exists a 3 -graph $H$ on $n+1$ vertices with minimum degree

$$
\left(\frac{19}{27}+O\left(\frac{1}{n}\right)\right)\left(\begin{array}{c}
n-1 \\
2
\end{array}\right)
$$

and no $K_{4}$-covering, establishing the desired lower bound on $c_{1}\left(K_{4}^{(3)}\right)$.

Proof of Theorem 1.3, upper bound. Set

$$
\alpha=\frac{19}{27}+7.4 \times 10^{-9} .
$$

By Proposition 2.3, it is enough to show that if $G$ is an $n$-vertex graph with $t_{\max } \leqslant(1-\alpha+$ $o(1))\left(\begin{array}{c}n-1 \\ 2\end{array}\right)$, then $e(G) \leqslant(\alpha+o(1))\left(\begin{array}{c}n-1 \\ 2\end{array}\right)$. This is done in Proposition 3.8 in the next section via a simple flag algebra calculation. 


\subsection{The graph $C_{5}^{(3)}$}

\section{Theorem 2.5.}

$$
0.55 \ldots=\frac{5}{9} \leqslant c_{1}\left(C_{5}^{(3)}\right) \leqslant 2-\sqrt{2}=0.58 \ldots
$$

Proof of Theorem 2.5, lower bound. We construct a 3-graph on $[3 n+1]$ as follows. Set aside $v_{\star}=3 n+1$, and partition the remaining vertices into an $n$-set $A$ and a $2 n$-set $B$. Let $H$ be the 3 graph on $[3 n+1]$ obtained by setting the link graph of $v_{\star}$ to be the union of a clique on $A$ and a clique on $B$, and adding all triples of the form $A A B$ or $A B B$. Every path of length 3 in the link graph of $v_{\star}$ gives rise to an independent set in $H$, hence there is no copy of the strong 5-cycle $C_{5}$ covering $v_{\star}$ in $H$. The degree of $v_{\star}$ in $H$ is

$$
\left(\begin{array}{l}
n \\
2
\end{array}\right)+\left(\begin{array}{c}
2 n \\
2
\end{array}\right)=\frac{5}{9}\left(\begin{array}{c}
3 n \\
2
\end{array}\right)-\frac{2 n}{3}
$$

and the degrees in the rest of the graph are all at least

$$
\min \left((|A|-1)|B|+\left(\begin{array}{c}
|B| \\
2
\end{array}\right),(|B|-1)|A|+\left(\begin{array}{c}
|A| \\
2
\end{array}\right)\right)=n(2 n-1)+\left(\begin{array}{c}
n \\
2
\end{array}\right)=\frac{5}{9}\left(\begin{array}{c}
3 n \\
2
\end{array}\right)-\frac{2 n}{3} .
$$

Thus

$$
c_{1}\left(3 n+1, C_{5}\right) \geqslant \frac{5}{9}\left(\begin{array}{c}
3 n \\
2
\end{array}\right)-\frac{2 n}{3}
$$

as desired.

Proof of Theorem 2.5, upper bound. Mubayi and Rödl [50, Theorem 1.9] proved $\pi\left(C_{5}^{(3)}\right) \leqslant 2-$ $\sqrt{2}$. An easy modification of their proof shows that $\alpha=2-\sqrt{2}$ is in fact an upper bound for the covering threshold. Indeed, let $H$ be a 3-graph on $n$ vertices with $\delta_{1}(H) \geqslant \alpha\left(\begin{array}{c}n-1 \\ 2\end{array}\right)+c(2 n-1)$, for some $c \geqslant 10$. Let $x$ be an arbitrary vertex in $V(H)$. By averaging, there exists $y \in V(H)$ such that $\operatorname{deg}(x y) \geqslant \alpha n$. Form the multigraph $G=H_{x} \cup H_{y}$ as in [50, proof of Theorem 1.9, p. 151]. Then [50, Claim, p. 151] shows that if there is no copy of $C_{5}^{(3)}$ covering the pair $x y$, then $G$ satisfies the conditions of [50, Lemma 6.2, p. 149], and one can conclude as Mubayi and Rödl do that one of $x$ and $y$ has degree at most $\alpha\left(\begin{array}{c}n-1 \\ 2\end{array}\right)+c(2 n-1)-n$ in $H$, contradicting our minimum degree assumption.

2.5 The graphs $K_{t}^{(3)}, t \geqslant 5$

Proposition 2.6. For all $t \geqslant 4$,

$$
c_{1}\left(K_{t+1}^{(3)}\right) \leqslant \frac{-1+\sqrt{3-2 c_{1}\left(K_{t}^{(3)}\right)}}{1-c_{1}\left(K_{t}^{(3)}\right)} .
$$

Proof. Let $\varepsilon>0$ and $n$ be sufficiently large. Suppose that $H$ is a 3-graph on $n$ vertices with $\delta_{1}(H) \geqslant$ $\alpha\left(n^{2} / 2\right)$ for some $\alpha>0$ satisfying

$$
1+\frac{2}{\alpha}-\frac{2}{\alpha^{2}}=c_{1}\left(K_{t}^{(3)}\right)+\varepsilon
$$

Let $v_{\star}$ be an arbitrary vertex. By averaging, there exists a vertex $x \in V \backslash\left\{v_{\star}\right\}$ and an $\alpha n$-set $V^{\prime}$ such that $V^{\prime} \subseteq \Gamma\left(x, v_{\star}\right)$. Observe that

$$
e\left(H_{x}\left[V^{\prime}\right]\right) \geqslant e\left(H_{x}\right)-\left|V^{\prime}\right|\left(n-\left|V^{\prime}\right|\right)-\left(\begin{array}{c}
n-\left|V^{\prime}\right| \\
2
\end{array}\right)
$$


and an analogous bound holds for $e\left(H_{v_{\star}}\left[V^{\prime}\right]\right)$. Thus

$$
\begin{aligned}
e\left(H_{x}\left[V^{\prime}\right] \cap H_{v_{\star}}\left[V^{\prime}\right]\right) & \geqslant e\left(H_{x}\right)+e\left(H_{v_{\star}}\right)-2\left|V^{\prime}\right|\left(n-\left|V^{\prime}\right|\right)-2\left(\begin{array}{c}
n-\left|V^{\prime}\right| \\
2
\end{array}\right)-\left(\begin{array}{c}
\left|V^{\prime}\right| \\
2
\end{array}\right) \\
& \geqslant\left(\alpha^{2}+2 \alpha-2\right) \frac{n^{2}}{2}+O(n) .
\end{aligned}
$$

On the other hand, for any $y \in V^{\prime}$, we have

$$
\begin{aligned}
\left|\Gamma(y) \cap\left(V^{\prime} \cup\left\{x, v_{\star}\right\}\right)^{(2)}\right| & \geqslant \operatorname{deg}(y)-\left(\left|V^{\prime}\right|+1\right)\left(n-\left|V^{\prime}\right|-2\right)-\left(\begin{array}{c}
n-\left|V^{\prime}\right|-2 \\
2
\end{array}\right) \\
& \geqslant\left(\alpha^{2}+\alpha-1\right) \frac{n^{2}}{2}+O(n) .
\end{aligned}
$$

Note that

$$
\min \left(\alpha^{2}+2 \alpha-2, \alpha^{2}+\alpha-1\right)=\alpha^{2}+2 \alpha-2=\left(c_{1}\left(K_{t}^{(3)}\right)+\varepsilon\right) \alpha^{2} .
$$

Let $H^{\prime}$ be the 3-graph obtained by taking $H\left[V^{\prime}\right]$ and adding a new vertex $z$ whose link graph consists precisely of those pairs $y y^{\prime} \in E\left(H_{x}\left[V^{\prime}\right] \cap H_{v_{\star}}\left[V^{\prime}\right]\right)$. By (2.6), (2.7) and (2.8),

$$
\delta_{1}\left(H^{\prime}\right) \geqslant\left(c_{1}\left(K_{t}\right)+\frac{\varepsilon}{2}\right)\left(\begin{array}{c}
v\left(H^{\prime}\right) \\
2
\end{array}\right) \text {. }
$$

Thus provided $\alpha n=v\left(H^{\prime}\right)$ is sufficiently large, there must be a set $S \subseteq V^{\prime}$ such that $S \cup\{z\}$ induces a copy of $K_{t}^{(3)}$ in $H^{\prime}$ covering $z$. But then by construction of $H^{\prime}$, this implies that $S \cup\left\{x, v_{\star}\right\}$ induces a copy of $K_{t+1}^{(3)}$ covering $v_{\star}$ in $H$. It follows that $\alpha \geqslant c_{1}\left(K_{t+1}^{(3)}\right)$, and hence (since $\varepsilon>0$ was arbitrary) that

$$
c_{1}\left(K_{t+1}^{(3)}\right) \leqslant \frac{-1+\sqrt{3-2 c_{1}\left(K_{t}^{(3)}\right)}}{1-c_{1}\left(K_{t}^{(3)}\right)} .
$$

Proposition 2.7. Suppose there exists a 3-graph $H$ on $[N]$ such that

(i) every vertex of $H$ has degree at most $d$,

(ii) every $t$-set of vertices from $V(H)$ spans at least one edge.

Then we have

$$
c_{1}\left(K_{t+1}^{(3)}\right) \geqslant \min \left(1-\frac{1}{N}, 1-\frac{2 d}{N^{2}}\right)
$$

Proof. We construct a 3-graph $G$ on $[N n+1]$ as follows. Set aside $v_{\star}=N n+1$, and partition the remaining vertices into $n$-sets $V_{1}, V_{2}, \ldots, V_{N}$. Now let the link graph of $v_{\star}$ in $G$ be the complete $N$-partite graph on $[N n]$ with partition $\sqcup_{i=1}^{N} V_{i}$. To make up the remainder of the edges of $G$, add in all triples $v_{1} v_{2} v_{3}$ from $[\mathrm{Nn}]^{(3)}$ with $v_{j} \in V_{i_{j}}$ for $j=1,2,3$ and $i_{1} i_{2} i_{3} \notin E(H)$.

Clearly

$$
\operatorname{deg}_{G}\left(v_{\star}\right)=\left(\begin{array}{c}
n N \\
2
\end{array}\right)-N\left(\begin{array}{l}
n \\
2
\end{array}\right)=\left(1-\frac{1}{N}\right)\left(\begin{array}{c}
n N \\
2
\end{array}\right)+O(n),
$$

and every other vertex $x \in[n N]$ with $x \in V_{i}$ has degree

$$
\operatorname{deg}_{G}(x)=n(N-1)+\left(\begin{array}{c}
n N-1 \\
2
\end{array}\right)-\operatorname{deg}_{H}(i) n^{2} \geqslant\left(1-\frac{2 d}{N^{2}}\right)\left(\begin{array}{c}
n N \\
2
\end{array}\right)+O(n)
$$


Thus

$$
\delta_{1}(G) \geqslant \min \left(1-\frac{1}{N}, 1-\frac{2 d}{N^{2}}\right)\left(\begin{array}{c}
n N \\
2
\end{array}\right)+O(n) .
$$

Furthermore, every complete graph $G_{v_{\star}}[T]$ on $|T|=t$ vertices in the link graph of $v_{\star}$ in $G$ meets $t$ different parts $V_{i_{1}}, \ldots, V_{i_{t}}$ from our partition of $[n N]$. By assumption, $i_{1} i_{2}, \ldots, i_{t}$ spans at least one edge of $H$, whence we have that at least one of the triples from $T^{(3)}$ is missing from $E(G)$. In particular, $\left\{v_{\star}\right\} \cup T$ does not span a copy of $K_{t+1}^{(3)}$ in $G$, and $G$ fails to have a $K_{t+1}^{(3)}$-cover. The proposition follows.

A natural family of 3-graphs for applications of Proposition 2.7 are Steiner triple systems (STS), where each pair of vertices is contained in a unique edge. Let $\alpha_{t}$ denote the minimum of the independence number over all STS of order $t$. The unique (up to isomorphism) STS of orders 3 and 7 are the 3-edge $K_{3}^{(3)}$ and the Fano plane $S_{7}$ respectively, which give $\alpha_{3}=2, \alpha_{7}=4$. The affine plane of order $9, S_{9}$, is the unique up to isomorphism STS of order 9 and has $\alpha\left(S_{9}\right)=\alpha_{9}=4$. It is further known that $\alpha_{13}=6, \alpha_{15}=6$ [48], and $\alpha_{19}=7$ [8] (see also the monograph of Kaski and Östergård [33]).

\section{Proposition 2.8.}

$$
\begin{aligned}
& 0.8888 \ldots=\frac{8}{9} \leqslant c_{1}\left(K_{6}^{(3)}\right) \leqslant 0.947962 \ldots, \\
& 0.9333 \ldots=\frac{14}{15} \leqslant c_{1}\left(K_{8}^{(3)}\right) \leqslant 0.98793 \ldots, \\
& 0.9473 \ldots=\frac{18}{19} \leqslant c_{1}\left(K_{9}^{(3)}\right) \leqslant 0.99404 \ldots
\end{aligned}
$$

Proof of Proposition 2.8, lower bound. Apply Proposition 2.7 to STS of orders 9, 15 and 19 with minimum independence numbers, and observe that an STS of order $t$ is a $d=(t-1) / 2$-regular 3-graph, so that

$$
\min \left(1-\frac{1}{t}, 1-\frac{2 d}{t^{2}}\right)=1-\frac{1}{t}
$$

Proof of Proposition 2.8, upper bound. Repeatedly apply Proposition 2.6 with our upper bound $c_{1}\left(K_{4}\right) \leqslant 19 / 27+7.4 \times 10^{-9}$ from Theorem 1.3.

Remark. The lower bounds on the covering densities in Proposition 2.8 above are strictly stronger than the bounds one gets from the conjectured values of the corresponding Turán densities.

In each case they are about $5 \times 10^{-2}$ below our upper bounds. Note that if one applies Proposition 2.7 to the unique STS on 3 -vertices, one gets a lower bound of $2 / 3$ for $c_{1}\left(K_{4}^{(3)}\right)$. We obtained an improvement of this bound in Theorem 1.3 by almost $5 \times 10^{-2}$ by adding a few edges in the link graph of $v_{\star}$ and deleting a few triples meeting the corresponding pairs. It seems natural to believe that a similar (albeit significantly more intricate) process would similarly improve the lower bounds in Proposition 2.8. If we had to guess, we would thus say that the true value of $c_{1}\left(K_{t}^{(3)}\right)$ for $t=6,8,9$ probably lies closer to the upper bounds we give.

For completeness, we give (very weak) bounds on $c_{1}\left(K_{5}^{(3)}\right)$, which show $c_{1}\left(K_{4}^{(3)}\right)<c_{1}\left(K_{5}^{(3)}\right)<$ $c_{1}\left(K_{6}^{(3)}\right)$. 


\section{Proposition 2.9.}

$$
\frac{3}{4} \leqslant c_{1}\left(K_{5}^{(3)}\right) \leqslant 0.8842 \ldots
$$

Proof of Proposition 2.9, lower bound. Consider a partition of [2n] into $n$-sets, $[2 n]=V_{1} \sqcup V_{2}$. Let $G$ be the 3-graph on [2n] whose edge-set consists of all triples meeting both $V_{1}$ and $V_{2}$. It is easily checked that $G$ is $K_{5}^{(3)}$-free and has minimum degree

$$
\left(\begin{array}{c}
2 n-1 \\
2
\end{array}\right)-\left(\begin{array}{c}
n-1 \\
2
\end{array}\right)=\frac{3}{4}\left(\begin{array}{c}
2 n-1 \\
2
\end{array}\right)+O(n),
$$

giving us the required lower bound.

Proof of Proposition 2.9, upper bound. Apply Proposition 2.6 with our upper bound

$$
c_{1}\left(K_{4}^{(3)}\right) \leqslant \frac{19}{27}+7.4 \times 10^{-9}
$$

from Theorem 1.3.

\section{Triangle-degree in graphs}

In this section we investigate the problem of minimizing the maximum triangle-degree $\tau(\rho) n^{2} / 2$ in a 2-graph with a given edge density $\rho$. We give upper bound constructions for $\tau(\rho)$, which we conjecture are best possible. We show that our conjecture holds for tripartite graphs and use flag algebra computations to bound below $\tau(\rho)$ for general graphs with $1 / 2<\rho \leqslant 2 / 3$.

\subsection{Proof of Theorem 1.4}

Proposition 3.1. Conjecture 1.7 implies $c_{1}\left(K_{4}^{(3)}\right)=19 / 27$.

Proof. Suppose $\rho=c_{1}\left(K_{4}^{(3)}\right)$. By Proposition 2.3, there exists a sequence $\left(G_{n}\right)_{n \in \mathbb{N}}$ of 2-graphs with $v\left(G_{n}\right) \rightarrow \infty, \rho\left(G_{n}\right) \geqslant \rho+o(1)$ and $t_{\max }\left(G_{n}\right) \leqslant(1-\rho+o(1))\left({ }^{v\left(G_{n}\right)-1}\right)$. In particular, this implies that $\tau(\rho) \leqslant 1-\rho$. If Conjecture 1.7 is true, then since $19 / 27 \in(2 / 3,3 / 4)$ we have

$$
\tau\left(\frac{19}{27}\right)=\frac{8}{27} \text { and } \tau\left(\frac{19}{27}+\varepsilon\right)>\frac{8}{27}
$$

for sufficiently small $\varepsilon>0$. Hence $\rho \leqslant 19 / 27$. Together with the lower bound from Theorem 1.3, we conclude that $c_{1}\left(K_{4}^{(3)}\right)=19 / 27$.

We now give constructions for two families of graphs used in the proof of Theorem 1.4.

Construction 3.2. (lower interval construction). Let

$$
\rho \in\left[\frac{r-1}{r}, \frac{r}{r+1}-\frac{1}{3 r(r+1)}\right] \text { for some } r \in \mathbb{N} .
$$

Suppose $n \in \mathbb{N}$ is divisible by $2 r$. Consider a balanced complete $r$-partite graph on $[n]$ with parts $V_{1}, \ldots, V_{r}$. Add inside each $V_{i}$ an arbitrary d-regular triangle-free graph $H_{i}$, where

$$
d=\left\lfloor\left(\rho-\frac{r-1}{r}\right) n\right\rfloor \text {. }
$$


Such triangle-free graphs exist since

$$
d \leqslant \frac{2}{3(r+1)} \frac{n}{r}
$$

(by our upper bound on $\rho$ ), which is less than $n /(2 r)$ (so one could take $H_{i}$ to be a balanced bipartite graph, for example). The resulting graph is $\lfloor\rho n\rfloor$-regular. We let $\mathcal{G}_{\rho, n}^{u}$ denote the family of all graphs that can be constructed in this way.

Construction 3.3. (upper interval construction). Let

$$
\rho \in\left[\frac{r}{r+1}-\frac{1}{3 r(r+1)}, \frac{r}{r+1}\right] \text { for some } r \in \mathbb{N} .
$$

Suppose $n \in \mathbb{N}$ is divisible by $2(r+1)$. Consider a balanced complete $(r+1)$-partite graph on $[n]$ with parts $V_{1}, \ldots, V_{r+1}$. Equally divide each $V_{i}$ into $V_{i}^{\prime}$ and $V_{i}^{\prime \prime}$. Let $\phi:[r+1] \rightarrow[r+1]$ be any bijection with the property that $\phi(i) \neq i$ for all $i \in[r+1]$ (any permutation of $[r+1]$ with no fixed point will do). Now, for every $i \in[r+1]$, replace the complete bipartite graph between $V_{i}^{\prime}$ and $V_{\phi(i)}^{\prime \prime}$ with an arbitrary d-regular bipartite subgraph $\mathrm{H}_{i}$, where

$$
d=\left\lceil\left(\rho-\frac{r}{r+1}+\frac{1}{2(r+1)}\right) n\right\rceil .
$$

The resulting graph is $\lceil\rho n\rceil$-regular. We let $\mathcal{G}_{\rho, n}^{d}$ denote the family of all graphs that can be constructed in this way.

Remark. The choices of the graphs $H_{i}$ in both Construction 3.2 and 3.3 give rise to very different graphs (lying at edit distance $\Omega\left(n^{2}\right)$ from each other). In particular, if Conjecture 1.7 is correct then the problem of minimizing the maximum triangle-degree is not stable. This stands in some contrast to the Rademacher-Turán problem for triangles, for which Pikhurko and Razborov [54] obtained a stability result, establishing that there is an asymptotically unique way of minimizing the number of triangles for a given edge density. This instability is observed even at the level of subgraph frequencies; for example, in the first construction we could take as $H_{i}$ a subgraph of a blow-up of the five-cycle instead of a bipartite graph, provided

$$
\rho \leqslant \frac{r-1}{r}+\frac{2}{5 r}
$$

In particular, this suggests that Conjecture 1.7 may be harder to resolve than the RademacherTurán problem for triangles, and might not amenable to standard flag algebraic approaches due to the instability of the extremal examples.

Proof of Theorem 1.4. Assume that

$$
\rho \in\left[\frac{r-1}{r}, \frac{r}{r+1}\right] \text { for some } r \in \mathbb{N} .
$$

When $r=1$, a $\rho n$-regular bipartite graph on $n$ vertices (we may use $\mathcal{G}_{\rho, n}^{u}$ and $\mathcal{G}_{\rho, n}^{d}$ as well) shows that $\tau(\rho)=0$. So we may assume that $r \geqslant 2$.

First assume that

$$
\rho \in\left[\frac{r-1}{r}, \frac{r}{r+1}-\frac{1}{3 r(r+1)}\right] .
$$


Consider an arbitrary graph $G$ of $\mathcal{G}_{\rho, n}^{u}$, for some $n$ divisible by $2 r$. Pick a vertex $x \in V_{i}$. Let us compute the triangle-degree of $x$. There are at most $\left(n-\left|V_{i}\right|\right) d$ pairs $\left(y, x^{\prime}\right)$ with $x^{\prime} \in V_{i} \backslash\{x\}$, $y \in[n] \backslash V_{i}$ and $x x^{\prime} y$ forming a triangle in $G$. Further, there are at most

$$
\frac{1}{2} \sum_{j \neq i}\left|V_{j}\right| d
$$

pairs $\left(y, y^{\prime}\right)$ with $y, y^{\prime} \in V_{j} \neq V_{i}$ and $x y y^{\prime}$ forming a triangle in $G$. Finally, there are at most

$$
\frac{1}{2} \sum_{j: j \neq i} \sum_{k: k \neq i, j}\left|V_{j}\right|\left|V_{k}\right|
$$

pairs $(y, z)$ with $y \in V_{j}, z \in V_{k}, V_{i}, V_{j}, V_{k}$ all distinct and $x y z$ forming a triangle in $G$. Since each part $V_{i}$ is triangle-free by construction, there are no other triangles in $G$ containing $x$, and the triangle-degree of $x$ is thus at most

$$
\begin{aligned}
t_{G}(x) & =\frac{r-1}{r} n\left\lfloor\left(\rho-\frac{r-1}{r}\right) n\right\rfloor+\frac{r-1}{2 r} n\left\lfloor\left(\rho-\frac{r-1}{r}\right) n\right\rfloor+\frac{(r-1)(r-2)}{2 r^{2}} n^{2} \\
& =\left(\frac{(r-1)(r-2)}{r^{2}}+\frac{3(r-1)}{r}\left(\rho-\frac{r-1}{r}\right)\right) \frac{n^{2}}{2}+O(n) .
\end{aligned}
$$

This gives the claimed upper bound on $\tau(\rho)$ for

$$
\rho \in\left[\frac{r-1}{r}, \frac{r}{r+1}-\frac{1}{3 r(r+1)}\right] .
$$

Next, assume that

$$
\rho \in\left[\frac{r}{r+1}-\frac{1}{3 r(r+1)}, \frac{r}{r+1}\right] .
$$

Consider an arbitrary graph $G$ of $\mathcal{G}_{\rho, n}^{d}$, for some $n$ divisible by $2(r+1)$. Pick a vertex $x \in V_{i}^{\prime}$ (the case when $x \in V_{i}^{\prime \prime}$ is analogous). When computing the triangle-degree of $x$, it is more convenient to count the number of triangles containing $x$ in the balanced complete $(r+1)$-partite graph from which an edge was deleted when constructing $G$. Observe that every triangle has lost at most one edge.

First of all, we have lost

$$
\left(\left|V_{\phi(i)}^{\prime \prime}\right|-d\right)\left(\frac{r-1}{r+1}\right) n
$$

triangles of the form $x y z$ with $y \in V_{\phi(i)}^{\prime \prime}$. Secondly, for every

$$
y \in[n] \backslash\left(V_{i} \cup V_{\phi(i)}^{\prime \prime} \cup V_{\phi^{-1}(i)}^{\prime}\right),
$$

there are $n /(2(r+1))-d$ vertices

$$
z \in[n] \backslash\left(V_{i} \cup V_{\phi(i)}^{\prime \prime} \cup V_{\phi^{-1}(i)}^{\prime}\right)
$$

such that the edge $y z$ was lost. This results in

$$
\frac{r-1}{r+1} \frac{n}{2}\left(\frac{n}{2(r+1)}-d\right)
$$


lost triangles $x y z$. In total there are

$$
\begin{aligned}
& \left(\frac{n}{2(r+1)}-d\right)\left(\frac{r-1}{r+1}\right) n+\frac{n}{2}\left(\frac{r-1}{r+1}\right)\left(\frac{n}{2(r+1)}-d\right) \\
& =3\left(\frac{r-1}{r+1}\right)\left(\frac{r}{r+1}-\rho\right) \frac{n^{2}}{2}+O(n)
\end{aligned}
$$

lost triangles for $x$. Subtracting this quantity from the triangle-degree of $x$ in the original complete balanced $(r+1)$-partite graph, we get

$$
t_{G}(x)=\left(\frac{r(r-1)}{(r+1)^{2}}-3\left(\frac{r-1}{r+1}\right)\left(\frac{r}{r+1}-\rho\right)\right) \frac{n^{2}}{2}+O(n) .
$$

This gives the claimed upper bound on $\tau(\rho)$ for

$$
\rho \in\left[\frac{r}{r+1}-\frac{1}{3 r(r+1)}, \frac{r}{r+1}\right] \text {. }
$$

\subsection{Proof of Theorem 1.8}

For this range of $e(G)$, Conjecture 1.7 states that, for any $n$-vertex graph $G$,

$$
t_{\max }(G) \geqslant \begin{cases}0 & \text { if } e(G) \leqslant \frac{n^{2}}{4}, \\ \frac{3}{2}\left(e(G)-\frac{n^{2}}{4}\right)+O(n) & \text { if } \frac{n^{2}}{4} \leqslant e(G) \leqslant \frac{11}{36} n^{2}, \\ e(G)-\frac{2}{9} n^{2}+O(n) & \text { if } \frac{11}{36} n^{2} \leqslant e(G) \leqslant \frac{1}{3} n^{2} .\end{cases}
$$

Remark. Since $3 / 10<11 / 36$ and since for $e(G)<(11 / 36) n^{2}$ we have

$$
e(G)-\frac{2}{9} n^{2}>\frac{3}{2}\left(e(G)-\frac{n^{2}}{4}\right)
$$

Theorem 1.8 implies that Conjecture 1.7 holds true for all tripartite graphs.

Proof of Theorem 1.8. Let $G$ be an $n$-vertex tripartite graph with partition $A \sqcup B \sqcup C$. Since $t_{\max }(G)$ is non-negative, we only need to consider the case when $e(G)>n^{2} / 4$. Assume without loss of generality that

$$
|A| \geqslant|B| \geqslant|C| \text {. }
$$

Suppose $|A|=x n$ and $|B|=y n$ (and so $|C|=(1-x-y) n$ ). Then $x \geqslant y \geqslant(1-x) / 2 \geqslant 0$, and in particular $x \geqslant 1 / 3$. Since

$$
|B||C| \leqslant\left(\frac{1-x}{2}\right)^{2}
$$

we have

$$
e(G) \leqslant|A|(n-|A|)+|B||C| \leqslant\left(x(1-x)+\left(\frac{1-x}{2}\right)^{2}\right) n^{2} .
$$

The function of $x$ on the right-hand side has derivative $3 / 2(1 / 3-x) n^{2} \leqslant 0$ for $x \geqslant 1 / 3$, and attains the value $n^{2} / 4$ at $x=2 / 3$. Since $e(G)>n^{2} / 4$, we must have $x<2 / 3$. 
Write $\alpha$ for the edge density of $G$ between parts $B$ and $C, \beta$ for the edge density between parts $A$ and $C$, and $\gamma$ for the edge density between parts $A$ and $B$. So we have

$$
\frac{e(G)}{n^{2}}=\gamma x y+\beta x(1-x-y)+\alpha y(1-x-y) .
$$

Since $x \geqslant y \geqslant 1-x-y$, if $\alpha+\beta+\gamma=S \leqslant 2$ then $e(G) / n^{2}$ is maximized by letting $\gamma=\min (S, 1)$, $\beta=S-\gamma$ and $\alpha=0$, i.e. by making $G$ bipartite. But a bipartite graph contains at most $n^{2} / 4$ edges, contradicting our lower bound on $e(G)$. Thus we assume $\alpha+\beta+\gamma=2+s$ for some $s$ with $0<$ $s \leqslant 1$. Further, if $x, s$ are fixed with $x \geqslant y \geqslant 1-x-y$, then $e(G) / n^{2}$ is maximized by letting $\gamma=1$, $\beta=1, \alpha=s$ and $y=(1-x) / 2$. In other words, we have

$$
\frac{e(G)}{n^{2}} \leqslant f_{1}(x, s):=x-x^{2}+\frac{s}{4}(1-x)^{2} .
$$

Since

$$
\frac{\partial}{\partial x} f_{1}(x, s)=1-2 x-\frac{s}{2}(1-x)=\left(\frac{2-s}{2}\right)-\left(\frac{4-s}{2}\right) x,
$$

when $s$ is fixed, $f_{1}(x, s)$ attains a maximum at

$$
x_{\star}=\frac{2-s}{4-s} \in\left[\frac{1}{3}, \frac{1}{2}\right] \quad(\text { as } 0 \leqslant s \leqslant 1) .
$$

Consequently,

$$
\frac{e(G)}{n^{2}} \leqslant f_{1}(x, s) \leqslant f_{1}\left(x_{\star}, s\right)=\frac{1}{4-s} .
$$

On the other hand, we can give a lower bound on $t_{\max }(G) / n^{2}$ as follows. Select vertices $a \in A$, $b \in B$ and $c \in C$ uniformly at random. By the union bound,

$$
\begin{aligned}
\mathbb{P}(a b c \text { induces a triangle }) & \geqslant \mathbb{P}(a b \in E(G))-\mathbb{P}(b c \notin E(G))-\mathbb{P}(a c \notin E(G)) \\
& =\alpha+\beta+\gamma-2 \\
& =s .
\end{aligned}
$$

In particular, $G$ must contain at least $s x y(1-x-y) n^{3}$ triangles. By averaging over all vertices $c \in C$, we have

$$
\frac{t_{\max }(G)}{n^{2}} \geqslant \frac{s x y(1-x-y) n^{3}}{|C| n^{2}}=s x y .
$$

Since $x \geqslant y \geqslant 1-x-y$, for fixed $s$ and $x, s x y$ is minimized by setting $y=(1-x) / 2$. Thus

$$
\frac{t_{\max }(G)}{n^{2}} \geqslant f_{2}(x, s):=\frac{s x(1-x)}{2} .
$$

Having done this preparatory work, we can now prove the theorem by using the following claim.

\section{Claim 3.4.}

$$
t_{\max }(G) \geqslant \begin{cases}e(G)-\frac{2}{9} n^{2} & \text { if } s \geqslant \frac{2}{3} \\ \frac{3}{2}\left(e(G)-\frac{n^{2}}{4}\right) & \text { if } s<\frac{2}{3}\end{cases}
$$


To see why Claim 3.4 implies Theorem 1.8, first assume $e(G) \geqslant(3 / 10) n^{2}$. By (3.2), we have $s \geqslant 2 / 3$. Then Claim 3.4 gives that $t_{\max }(G) \geqslant e(G)-(2 / 9) n^{2}$. Now assume $e(G)<(3 / 10) n^{2}$. If we still have $s \geqslant 2 / 3$, then by Claim 3.4,

$$
t_{\max }(G) \geqslant e(G)-\frac{2}{9} n^{2}>\frac{3}{2}\left(e(G)-\frac{n^{2}}{4}\right)
$$

because $e(G)<(11 / 36) n^{2}$. Otherwise $s<2 / 3$ and Claim 3.4 implies that

$$
t_{\max }(G) \geqslant \frac{3}{2}\left(e(G)-\frac{n^{2}}{4}\right),
$$

as desired.

Proof of Claim 3.4, case $s \geqslant 2 / 3$. By inequalities (3.1) and (3.3), we have

$$
\frac{e(G)}{n^{2}}-\frac{t_{\max }(G)}{n^{2}} \leqslant f_{1}(x, s)-f_{2}(x, s)=x-x^{2}+\frac{s}{4}(1-x)^{2}-\frac{s}{2} x(1-x) .
$$

It is an easy exercise in calculus to show that as a function of $x \in(0,1)$, the right-hand side is maximized at

$$
x_{\star}=\frac{2-2 s}{4-3 s} \leqslant \frac{1}{3}
$$

(as $s \geqslant 2 / 3$ ), and is decreasing in $\left[x_{\star},+\infty\right)$. Under our assumption $x \geqslant 1 / 3$, we thus have

$$
f_{1}(x, s)-f_{2}(x, s) \leqslant f_{1}\left(\frac{1}{3}, s\right)-f_{2}\left(\frac{1}{3}, s\right)=\frac{2}{9} .
$$

This implies that $t_{\max }(G) \geqslant e(G)-(2 / 9) n^{2}$.

Proof of Claim 3.4, case $0<s<2 / 3$. By inequalities (3.1) and (3.3) we have

$$
\begin{aligned}
\frac{3}{2} \frac{e(G)}{n^{2}}-\frac{3}{8}-\frac{t_{\max }(G)}{n^{2}} & \leqslant \frac{3}{2} f_{1}(x, s)-\frac{3}{8}-f_{2}(x, s) \\
& =\frac{3}{2}\left(x(1-x)-\frac{1}{4}\right)+\frac{s}{8}(1-x)(3-7 x) .
\end{aligned}
$$

If $x \in[3 / 7,1]$, then both terms on the right-hand side are non-positive. Assume now that $x \in$ $[1 / 3,3 / 7)$. Then, for such values of $x$, the right-hand side is an increasing function of $s$. Applying our assumption on $s$, its value is at most

$$
\frac{3}{2} f_{1}\left(x, \frac{2}{3}\right)-\frac{3}{8}-f_{2}\left(x, \frac{2}{3}\right)=-\frac{1}{8}+\frac{2}{3} x-\frac{11}{12} x^{2} .
$$

The discriminant of this quadratic is

$$
\frac{4}{9}-4 \cdot \frac{1}{8} \cdot \frac{11}{12}=-\frac{1}{72}<0,
$$

so the expression above is (strictly) non-positive. We deduce that the right-hand side of (3.4) is non-positive for every value of $x \in[0,1]$. This yields

$$
t_{\max }(G) \geqslant \frac{3}{2}\left(e(G)-\frac{1}{4} n^{2}\right) \text {. }
$$




\subsection{Flag algebra bounds}

In this section we will employ Razborov's [55] flag algebra framework, and more specifically his semidefinite method, to obtain bounds for some of the problems we study. The semidefinite method has become a fairly standard tool in extremal combinatorics; see e.g. [57] for a survey of some of the early applications. As the method is well established and we have only obtained nonsharp bounds using it, we give only minimal details here, without expounding on the underlying theoretical machinery.

We have used Flagmatic to perform our flag algebra computations; this is an open source program written by Emil Vaughan and later developed further by Jakub Sliacan [62], who currently maintains a Flagmatic page on GitHub [62]. We have used Vaughan's Flagmatic 2.0 in this paper. We refer readers to [20] and to the Flagmatic 2.0 section on the web page [62] for a description of the inner workings of Flagmatic and download links for the program. Our calculations involve the use of flag inequalities given as 'axioms'. The use of such 'axioms' first appeared in [18], where an edge-maximization problem was solved subject to a codegree constraint. We refer readers interested in the details to either Section 3 in that paper or to the Flagmatic 2.0 web page [62].

Let $T_{1}$ denote the $([1], \varnothing)$-flag consisting of a triangle with one vertex labelled 1 . Let $\rho$ denote the $(\emptyset, \emptyset)$-flag consisting of a single 2 -edge (this flag corresponds to the edge density). Let $f(\rho)$ denote the upper bound on $\tau(\rho)$ given in Theorem 1.4.

The function $f(\rho)$ is piecewise linear, continuous and strictly increasing in the interval $(1 / 2,1]$. In particular, it has a piecewise linear inverse. Over any subinterval $I \subseteq[1 / 2,1]$ on which is $f$ is linear, we can use a semidefinite method to obtain an upper bound on how much $\tau(\rho)$ can deviate from $f(\rho)$ on $I$ by giving an upper bound for the following problem.

Problem 3.5. Maximize $\rho-f^{-1}(y)$ over $y \in f(I)$ subject to the constraint $T_{1} \leqslant y$.

Note that the constraint we have given corresponds to requiring that all but $o(1)$ of the vertices have triangle-degree at most $y\left(n^{2} / 2\right)+o(n)$ (which is slightly weaker than is required for $\tau)$. A standard flag algebra computation will give us an upper bound $\varepsilon_{I}>0$ on the solution to Problem 3.5. If $f(x)=a x+b$ over the interval $I$, then this tells us that $f\left(x-\varepsilon_{I}\right)=a\left(x-\varepsilon_{I}\right)+b$ is a lower bound for $\tau(x)$ on the interval $I-\varepsilon_{I}:=\left\{x \in I: x \leqslant \sup I-\varepsilon_{I}\right\}$, that is, $f(\rho)$ is at most $a \varepsilon_{I}$ away from the true value of $\tau(\rho)$ on $I-\varepsilon$. Using this technique, we obtain the following.

\section{Theorem 3.6.}

$$
\tau(\rho) \geqslant \begin{cases}f(\rho)-0.0010705 & \text { if } \rho \in[1 / 2,29 / 54], \\ f(\rho)-0.0044863 & \text { if } \rho \in[29 / 54,31 / 54], \\ f(\rho)-0.0106917 & \text { if } \rho \in[31 / 54,11 / 18], \\ f(\rho)-0.0106917 & \text { if } \rho \in[11 / 18,17 / 27], \\ f(\rho)-0.0058198 & \text { if } \rho \in[17 / 27,35 / 54], \\ f(\rho)-0.0002057 & \text { if } \rho \in[35 / 54,2 / 3], \\ f(\rho)-0.00123143 & \text { if } \rho \in[2 / 3,25 / 36], \\ f(\rho)-0.00534603 & \text { if } \rho \in[25 / 36,13 / 18], \\ f(\rho)-0.00534583 & \text { if } \rho \in[13 / 18,53 / 72], \\ f(\rho)-0.00189005 & \text { if } \rho \in[53 / 72,3 / 4] .\end{cases}
$$

Proof. The theorem follows from standard algebra computations using the method outlined above. Running the script theorem38. sage, which is found in the auxiliary files of this arXiv submission on Flagmatic 2.0, yields the bounds claimed above. (The resulting computation certificates are somewhat large, but the computation itself can easily be run on a modern laptop computer.) 
We also 'zoom in' on the value $\rho=\rho_{\star}$ at which $\tau(\rho)$ becomes greater than $1-\rho$, and which we conjecture is equal to $19 / 27$. This is done by giving an upper bound for the following variant of Problem 3.5.

Problem 3.7. Maximize $1-x-\rho$ subject to the constraint $T_{1} \leqslant x$.

Suppose for some fixed $x$ we perform a flag algebra calculation and get a non-positive upper bound for the solution to Problem 3.7. This implies that any $n$-vertex graph with at least $(1-x)\left(n^{2} / 2\right)+o\left(n^{2}\right)$ edges must have a positive proportion of its vertices having triangle-degree greater than $x\left(n^{2} / 2\right)+o\left(n^{2}\right)$. In particular, we must have $\rho_{\star} \leqslant x$. Using this technique, we obtain the following bounds on $\rho_{\star}$.

\section{Proposition 3.8 .}

$$
\rho_{\star} \leqslant \frac{19}{27}+7.4 \times 10^{-9}
$$

Proof. The theorem follows from standard algebra computations using the method outlined above. Running the script theorem310. sage, which is found in the auxiliary files of this arXiv submission on Flagmatic 2.0, yields the bounds claimed above. (This is a much smaller computation than the one required for Theorem 3.6.)

\section{Concluding remarks}

In earlier sections we showed that

$$
c_{1}\left(K_{4}^{(3-)}\right)=\frac{\sqrt{13}-1}{6}, \quad \frac{19}{27} \leqslant c_{1}\left(K_{4}^{(3)}\right) \leqslant \frac{19}{27}+7.4 \times 10^{-9} \quad \text { and } \quad \frac{5}{9} \leqslant c_{1}\left(C_{5}^{(3)}\right) \leqslant 2-\sqrt{2} .
$$

We conjecture that $c_{1}\left(K_{4}^{(3)}\right)=19 / 27$ and $c_{1}\left(C_{5}^{(3)}\right)=5 / 9$.

\subsection{Book numbers of graphs}

In Section 3 we investigated the following question. Let $G$ be a graph on $n$ vertices with $m>$ $\operatorname{ex}\left(n, K_{3}^{(2)}\right)$ edges. What is the largest $t$ such that $G$ must have some vertex contained in at least $t$ triangles? A different but equally natural question is to ask: What is the largest $b$ such that $G$ must have some edge contained in at least $b$ triangles? This is in fact a well-studied problem in graph theory.

Definition Let $G$ be a 2-graph, and $x y \in E(G)$. The book size of $x y$ in $G$ is $\operatorname{bk}(x y)=\mathrm{bk}_{G}(x y):=$ $|\Gamma(x) \cap \Gamma(y)|$, the number of triangles in $G$ containing the edge $x y$. The book number of $G$ is

$$
\operatorname{bk}(G):=\max \{\operatorname{bk}(x y): x y \in E(G)\} .
$$

The study of book numbers in graphs was initiated by Erdős in 1962 [14], and has attracted considerable attention in extremal graph theory and Ramsey theory. Set

$$
\beta(n, m):=\min \{\operatorname{bk}(G): v(G)=n, e(G)=m\}
$$

and

$$
\beta(x):=\inf _{n}\left\{\operatorname{bk}(G) / n: v(G)=n, e(G) \geqslant x\left(\begin{array}{l}
n \\
2
\end{array}\right)\right\} .
$$

Erdös conjectured that

$$
\beta\left(n, \operatorname{ex}\left(n, K_{3}^{(2)}\right)+1\right)>\frac{n}{6} .
$$


This was proved by Edwards [13] and independently by Khadžiivanov and Nikiforov [37]. Bollobás and Nikiforov [6] determined $\beta(n, m)$ exactly for infinitely many $m$ with $n^{2} / 4<m<$ $n^{2} / 3$.

A construction giving the best known lower bound on $\beta(n, m)$ was given by Erdös, Faudree and Györi [15], generalizing an earlier construction due to Erdös, Faudree and Rousseau [16].

Construction 4.1. (Erdös, Faudree and Györi [15]). Suppose $n=r_{1} \cdot r_{2} \cdot r_{3} \cdots r_{k-1} \cdot r_{k} t$, where $r_{1}, r_{2}, \ldots, r_{k}$, t are strictly positive integers satisfying $\left(r_{i-1}-1\right)^{2}<r_{i}$ for every $i \in[k]$. Set

$$
V=\left\{\left(i_{1}, i_{2}, \ldots, i_{k}, i_{k+1}\right): i_{j} \in\left[r_{j}\right] \text { for all } j \in[k], i_{k+1} \in[t]\right\} .
$$

Define a graph $G$ on $V$ by joining pairs of vectors from $V$ by an edge if and only if they differ in each of the first $k$ coordinates.

This construction gives rise to a $d$-regular graph with book number $b$, where

$$
d=\prod_{i=1}^{k}\left(\frac{r_{i}-1}{r_{i}}\right) n \text { and } b=\prod_{i=1}^{k}\left(\frac{r_{i}-2}{r_{i}}\right) n .
$$

Erdös, Faudree and Györi conjectured that this gives the correct behaviour for the minimum value of the book number in graphs subject to a minimum degree condition.

Conjecture 4.2. (Erdős, Faudree and Györi [15]). Let $x \in \mathbb{Q}$ with $1 / 2<x<1$. Let

$$
x=\prod_{i=1}^{k} \frac{r_{i}-1}{r_{i}}
$$

with $3 \leqslant r_{1}$ and $\left(r_{i-1}-1\right)^{2}<r_{i}$ for $2 \leqslant i \leqslant k$ be the (unique) 'greedy representation' of $x$. Set

$$
b(x)=\prod_{i=1}^{k} \frac{r_{i}-2}{r_{i}} .
$$

Then every graph on $n$ vertices with minimum degree $d \geqslant x n$ has book number at least $b(x) n$.

We believe that the minimum degree condition in Conjecture 4.2 can be replaced by a size condition, and this belief seemed to be borne out by flag algebra computations we ran for this problem.

Conjecture 4.3. Let $x \in \mathbb{Q} \cap(1 / 2,1)$ and $b(x)$ be as above. Then $\beta(x)=b(x)$, that is, any graph on $n$ vertices with at least $x\left(n^{2} / 2\right)$ edges has book number at least $b(x) n$.

\subsection{Maximal triangle-degree, book number and triangle density}

In Sections 3 and 4.1 we discussed the maximum triangle-degree of a vertex and the book number (i.e. maximum triangle-degree of an edge) in graphs, giving conjectures on their minimum value for a given edge density or minimum degree condition. Here we compare the conjectured behaviour of these two triangle-related extremal quantities with each other and with the minimal triangle density in graphs $G$ with $\rho\left(\begin{array}{c}n \\ 2\end{array}\right)$ edges for $1 / 2 \leqslant \rho \leqslant 2 / 3$.

Razborov [56] showed that such a graph $G$ contains at least $\kappa(\rho)\left(\begin{array}{l}n \\ 3\end{array}\right)+o\left(n^{3}\right)$ triangles, where

$$
\kappa(\rho)=\frac{1}{18}(1-\sqrt{2(2-3 \rho)})(2+\sqrt{2(2-3 \rho)})^{2} .
$$

In addition, Lo [42] showed that if the minimum degree of $G$ is at least $\rho n$, then it contains at least $\lambda(\rho)\left(\begin{array}{l}n \\ 3\end{array}\right)+o\left(n^{3}\right)$ triangles, where

$$
\lambda(\rho)=3 \rho(1-\rho)(2 \rho-1) .
$$




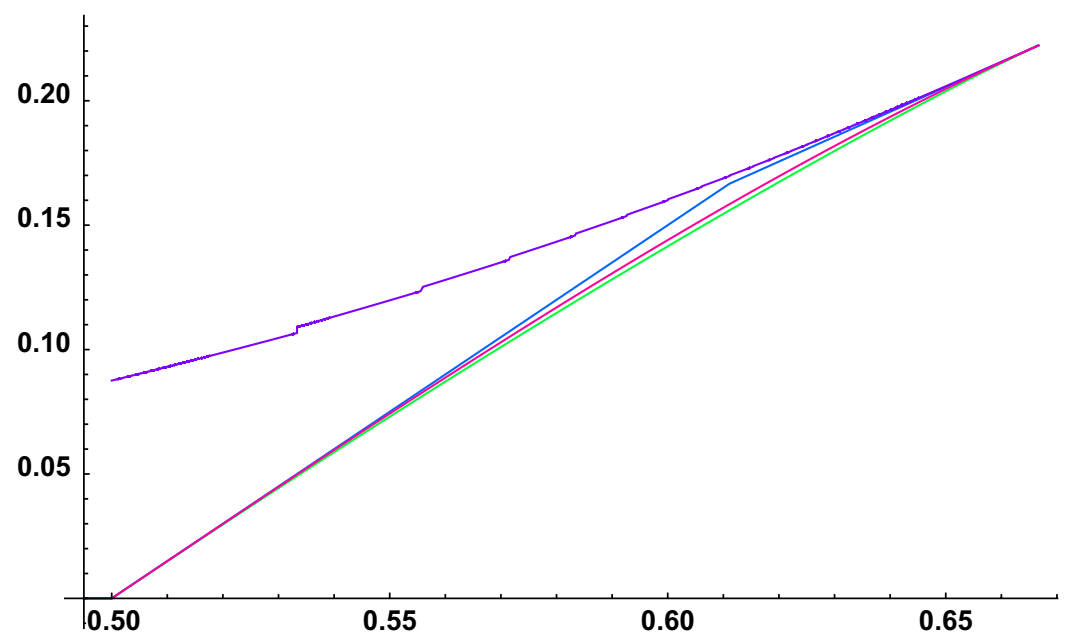

Figure 1. The functions $\rho \cdot \beta^{\prime}(\rho), \tau^{\prime}(\rho), \lambda(\rho), \kappa(\rho)$ (from top to bottom).

Conjecture 1.7 implies that every $n$-vertex graph with edge density $\rho$ contains a vertex with triangle-degree at least $\tau^{\prime}(\rho)\left(\begin{array}{l}n \\ 2\end{array}\right)+o\left(n^{2}\right)$, where

$$
\tau^{\prime}(\rho)= \begin{cases}\frac{3}{2}\left(\rho-\frac{1}{2}\right) & \text { if } \frac{1}{2} \leqslant \rho \leqslant \frac{11}{18}, \\ \rho-\frac{4}{9} & \text { if } \frac{11}{18} \leqslant \rho \leqslant \frac{2}{3} .\end{cases}
$$

Finally, let $\beta^{\prime}(x)$ denote the function obtained by extending the function $b(x)$ from Conjecture 4.2 from the rationals in $(1 / 2,2 / 3$ ] to a monotonically increasing left-continuous function on the whole interval. This last function unfortunately does not have a nice closed form, but we can plot an approximation of it (or rather, $\rho \beta^{\prime}(\rho)$ ) along the other three in Figure 1, allowing for a visual comparison of the four functions $\kappa, \lambda, \tau^{\prime}$ and $\rho \beta^{\prime}$ in the interval $\rho \in(1 / 2,2 / 3]$.

Clearly, by averaging we have that $\kappa(\rho)$ is the smallest of the functions in Figure 1. Assume now that Conjecture 1.7 is true. Then Constructions 3.2 and 3.3 provide $\rho n$-regular graphs $G$ of order $n$ with $t_{\max }(G)=\tau(\rho) n^{2} / 2+o(n)$. Averaging the triangle-degree over all vertices, this would give that $\lambda(\rho) \leqslant \tau(\rho)$.

Further assuming Conjecture 4.2 is true, Construction 4.1 gives a $\rho n$-regular graph $G$ of order $n$ with book number $\beta n$ that is triangle-degree regular with $t_{\max }(G)=\rho \beta(\rho)\left(\begin{array}{l}n \\ 2\end{array}\right)+o\left(n^{2}\right)$. This would imply that $\tau(\rho) \leqslant \rho \beta(\rho)$, and all together

$$
\kappa(\rho) \leqslant \lambda(\rho) \leqslant \tau(\rho) \leqslant \rho \beta(\rho) .
$$

In Figure 1 we have plotted the four functions $\kappa(\rho), \lambda(\rho), \tau^{\prime}(\rho)$ and $\rho \beta^{\prime}(\rho)$ in the interval $[1 / 2,2 / 3]$. As the plot shows, the inequalities in (4.1) with $\tau^{\prime}$ and $\beta^{\prime}$ taking the place of $\tau$ and $\beta$ all hold in $[1 / 2,2 / 3]$ with equality if and only if $\rho=1 / 2$ (for the first two inequalities) or $2 / 3$ (for all three).

\section{Acknowledgements}

The authors gratefully acknowledge the support of a Wenner-Gren guest professorship awarded to the third author to visit Umeå in May-June 2017, when we conducted this research. The first author would also like to thank the earlier support of an AMS-Simons grant for a visit to Atlanta in early 2016, in the course of which he and the third author began discussing vertex-degree 
thresholds for covering. Victor Falgas-Ravry's research is supported by VR grant 2016-03488. Klas Markström's research is supported by VR grant 2014-4897. Yi Zhao's research is supported by NSF grants DMS-1400073 and DMS-1700622.

\section{References}

[1] Andrásfai, B., Erdős, P. and Sós, V. T. (1974) On the connection between chromatic number, maximal clique and minimal degree of a graph. Discrete Math. 8 205-218.

[2] Baber, R., Johnson, J. R. and Talbot, J. (2010) The minimal density of triangles in tripartite graphs. LMS J. Comput. Math. $13388-413$.

[3] Baber, R. and Talbot, J. (2012) New Turán densities for 3-graphs. Electron. J. Combin. 19 1-21.

[4] Bollobás, B. (1974) Three-graphs without two triples whose symmetric difference is contained in a third. Discrete Math. 8 21-24.

[5] Bollobás, B. (1976) On complete subgraphs of different orders. Math. Proc. Cambridge Philos. Soc. 79 19-24.

[6] Bollobás, B. and Nikiforov, V. (2005) Books in graphs. Europ. J. Combin. 26 259-270.

[7] Bondy, A., Shen, J., Thomassé, S. and Thomassen, C. (2006) Density conditions for triangles in multipartite graphs. Combinatorica 26 121-131.

[8] Colbourn, C. J., Forbes, A. D., Grannell, M. J., Griggs, T. S., Kaski, P., Östergård, P. R. J., Pike, D. A. and Pottonen, O. (2010) Properties of the Steiner triple systems of order 19. Electron. J. Combin. 17 1-30.

[9] Czygrinow, A. (2016) Tight co-degree condition for packing of loose cycles in 3-graphs. J. Graph Theory 83 317-333.

[10] Czygrinow, A., DeBiasio, L. and Nagle, B. (2014) Tiling 3-uniform hypergraphs with $K_{4}^{3}-2 e$. J. Graph Theory $75124-$ 136.

[11] Czygrinow, A. and Nagle, B. (2001) A note on codegree problems for hypergraphs. Bull. Inst. Combin. Appl. 32 63-69.

[12] De Caen, D. and Füredi, Z. (2000) The maximum size of 3-uniform hypergraphs not containing a Fano plane. J. Combin. Theory Ser. B 78 274-276.

[13] Edwards, C. S. (1977) A lower bound for the largest number of triangles with a common edge. Unpublished manuscript.

[14] Erdős, P. (1962) On a theorem of Rademacher-Turán. Illinois J. Math 6 1-3.

[15] Erdős, P., Faudree, R. and Györi, E. (1995) On the book size of graphs with large minimum degree. Studia Sci. Math. Hungar. 30 25-46.

[16] Erdős, P., Faudree, R. J. and Rousseau, C. C. (1994) Extremal problems and generalized degrees. Discrete Math. 127 139-152.

[17] Erdős, P. and Stone, A. H. (1946) On the structure of linear graphs. Bull. Amer. Math. Soc 521.

[18] Falgas-Ravry, V., Marchant, E., Pikhurko, O. and Vaughan, E. R. (2015) The codegree threshold for 3-graphs with independent neighborhoods. SIAM J. Discrete Math. 29 1504-1539.

[19] Falgas-Ravry, V., Pikhurko, O., Vaughan, E. and Volec, J. (2017) The codegree threshold of $K_{4}^{-}$. Preprint.

[20] Falgas-Ravry, V. and Vaughan, E. R. (2013) Applications of the semi-definite method to the Turán density problem for 3-graphs. Combin. Probab. Comput. 22 21-54.

[21] Falgas-Ravry, V. and Zhao, Y. (2016) Codegree thresholds for covering 3-uniform hypergraphs. SIAM J. Discrete Math. 30 1899-1917.

[22] Fisher, D. C. (1989) Lower bounds on the number of triangles in a graph. J. Graph Theory 13 505-512.

[23] Füredi, Z. (1991) Turán type problems. In Surveys in Combinatorics 1991, Vol. 166 of London Mathematical Society Lecture Note Series, Cambridge University Press, pp. 253-300.

[24] Füredi, Z., Pikhurko, O. and Simonovits, M. (2005) On triple systems with independent neighbourhoods. Combin. Probab. Comput. 14 795-813.

[25] Gao, W. and Han, J. (2017) Minimum codegree threshold for $C_{6}^{3}$-factors in 3-uniform hypergraphs. Combin. Probab. Comput. 26 536-559.

[26] Gao, W., Han, J. and Zhao, Y. (2019) Codegree conditions for tiling complete $k$-partite $k$-graphs and loose cycles. Combin. Probab. Comput. 28 840-870. doi:10.1017/S096354831900021X

[27] Hajnal, A. and Szemerédi, E. (1970) Proof of a conjecture of Erdős. Combin. Theory Appl. 2 601-623.

[28] Hàn, H., Person, Y. and Schacht, M. (2009) On perfect matchings in uniform hypergraphs with large minimum vertex degree. SIAM J. Discrete Math 23 732-748.

[29] Han, J., Lo, A. and Sanhueza-Matamala, N. (2017) Covering and tiling hypergraphs with tight cycles. Electron. Notes Discret. Math. 61 561-567.

[30] Han, J., Lo, A., Treglown, A. and Zhao, Y. (2017) Exact minimum codegree threshold for $K_{4}^{-}$-factors. Combin. Probab. Comput. 26 856-885. doi:10.1017/S0963548317000268

[31] Han, J., Zang, C. and Zhao, Y. (2017) Minimum vertex degree thresholds for tiling complete 3-partite 3-graphs. J. Combin. Theory Ser. A 149 115-147.

[32] Han, J. and Zhao, Y. (2015) Minimum vertex degree threshold for $C_{4}^{3}$-tiling. J. Graph Theory 79 300-317. 
[33] Kaski, P. and Östergård, P. R. J. (2006) Classification Algorithms for Codes and Designs, Vol. 15 of Algorithms and Computation in Mathematics, Springer.

[34] Keevash, P. (2011) Hypergraph Turán problems. In Surveys in Combinatorics 2011, Vol. 392 of London Mathematical Society Lecture Note Series, Cambridge University Press, pp. 83-139.

[35] Keevash, P. and Mycroft, R. (2014) A Geometric Theory for Hypergraph Matching, Vol. 233, no. 1908, of Memoirs of the American Mathematical Society, American Mathematical Society.

[36] Keevash, P. and Zhao, Y. (2007) Codegree problems for projective geometries. J. Combin. Theory Ser. B 97 919-928.

[37] Khadžiivanov, N. and Nikiforov, V. (1979) Solution of a problem of P. Erdős about the maximum number of triangles with a common edge in a graph (in Russian). C.R. Acad. Bulgare Sci. 32 1315-1318.

[38] Khan, I. (2013) Perfect matchings in 3-uniform hypergraphs with large vertex degree. SIAM J. Discrete Math. 27 10211039.

[39] Kühn, D. and Osthus, D. (2006) Loose Hamilton cycles in 3-uniform hypergraphs of high minimum degree. J. Combin. Theory Ser. B 96 767-821.

[40] Kühn, D. and Osthus, D. (2009) The minimum degree threshold for perfect graph packings. Combinatorica 29 65-107.

[41] Kühn, D., Osthus, D. and Treglown, A. (2013) Matchings in 3-uniform hypergraphs. J. Combin. Theory Ser. B 103 291-305.

[42] Lo, A. (2012) Cliques in graphs with bounded minimum degree. Combin. Probab. Comput. 21 457-482.

[43] Lo, A. and Markström, K. (2013) Minimum codegree threshold for $\left(K_{4}-e\right)$-factors. J. Combin. Theory Ser. A 120 708-721.

[44] Lo, A. and Markström, K. (2014) $\ell$-degree Turán Density. SIAM J. Discrete Math. 28 1214-1225.

[45] Lo, A. and Markström, K. (2015) F-factors in hypergraphs via absorption. Graphs Combin. 31 679-712.

[46] Lovász, L. and Simonovits, M. (1976) On the number of complete subgraphs of a graph. In Proceedings of the Fifth British Combinatorial Conference (Aberdeen 1975), pp. 431-441.

[47] Lovász, L. and Simonovits, M. (1983) On the number of complete subgraphs of a graph II. In Studies in Pure Mathematics (P. Erdős et al., eds), Birkhäuser, pp. 459-495.

[48] Mathon, R. A., Phelps, K. T. and Rosa, A. (1982) Small Steiner triple systems and their properties. Ars Combin. 15 3-110.

[49] Mubayi, D. (2005) The co-degree density of the Fano plane. J. Combin. Theory Ser. B 95 333-337.

[50] Mubayi, D. and Rödl, V. (2002) On the Turán number of triple systems. J. Combin. Theory Ser. A $100136-152$.

[51] Mubayi, D. and Zhao, Y. (2007) Co-degree density of hypergraphs. J. Combin. Theory Ser. A 114 1118-1132.

[52] Mycroft, R. (2016) Packing k-partite k-uniform hypergraphs. J. Combin. Theory Ser. A 138 60-132.

[53] Nikiforov, V. (2011) The number of cliques in graphs of given order and size. Trans. Amer. Math. Soc. $3631599-1618$.

[54] Pikhurko, O. and Razborov, A. (2017) Asymptotic structure of graphs with the minimum number of triangles. Combin. Probab. Comput. 26 138-160.

[55] Razborov, A. (2007) Flag algebras. J. Symbolic Logic 72 1239-1282.

[56] Razborov, A. (2008) On the minimal density of triangles in graphs. Combin. Probab. Comput. 17 $603-618$.

[57] Razborov, A. A. (2013) Flag algebras: An interim report. In The Mathematics of Paul Erdős II (R. Graham et al., eds), Springer, pp. 207-232.

[58] Reiher, C. (2016) The clique density theorem. Ann. of Math. 184 683-707.

[59] Rödl, V. and Ruciński, A. (2010) Dirac-type questions for hypergraphs: A survey (or more problems for Endre to solve). In An Irregular Mind, Vol. 21 of Bolyai Society Mathematical Studies, Springer, pp. 561-590.

[60] Rödl, V., Ruciński, A. and Szemerédi, E. (2009) Perfect matchings in large uniform hypergraphs with large minimum collective degree. J. Combin. Theory Ser. A 116 613-636.

[61] Sidorenko, A. (1995) What we know and what we do not know about Turán numbers. Graphs Combin. 11 $179-199$.

[62] Sliacan, J. (2018) Flagmatic 2.0. https://github.com/jsliacan/flagmatic-2.0.

[63] Turán, P. (1941) On an extremal problem in graph theory (in Hungarian). Mat. Fiz. Lapok 48 436-452.

[64] Zhao, Y. (2016) Recent advances on Dirac-type problems for hypergraphs. In Recent Trends in Combinatorics, Vol. 159 of The IMA Volumes in Mathematics and its Applications, Springer, pp. 145-165.

Cite this article: Falgas-Ravry V, Markström K and Zhao Y (2021). Triangle-degrees in graphs and tetrahedron coverings in 3-graphs. Combinatorics, Probability and Computing 30, 175-199. https://doi.org/10.1017/S0963548320000061 